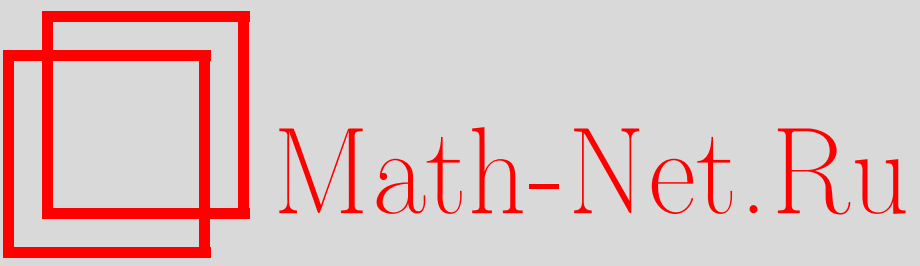

A. Basse-O'Connor, S.-E. Graversen, J. Pedersen, Stochastic integration on the real line, Теория вероятн. и ее примен., 2013, том 58, выпуск 2, 355-380

DOI: https://doi.org/10.4213/tvp4510

Использование Общероссийского математического портала Math-Net.Ru подразумевает, что вы прочитали и согласны с пользовательским соглашением

http://www.mathnet.ru/rus/agreement

Параметры загрузки:

IP: 54.210 .77 .194

26 апреля 2023 г., 14:54:37 


\title{
STOCHASTIC INTEGRATION ON THE REAL LINE
}

\begin{abstract}
Изучаются стохастические интегралы на предсказуемых $\sigma$ алгебрах относительно разностных семимартингалов и, более общим образом, относительно $\sigma$-конечных $L^{0}$-значных мер. Последние также называют формальными семимартингалами. В частности, мы вводим триплет $\sigma$-конечных мер и с его помощью характеризуем множество интегрируемых процессов. Особое внимание уделяется процессам Леви, индексированным вещественной прямой. У дивительным образом, в нашей ситуации отсутствуют многие основные свойства, выполненные в обычном случае $\mathbf{R}_{+}$. Полученные результаты позволяют определить и изучать различные классы стационарных процессов.
\end{abstract}

Ключевыле слова и фразыл: стохастический интеграл, (разностные) семимартингалы, процессы Леви, векторные меры.

1. Introduction. Let $(\Omega, \mathcal{F}, \mathbf{P})$ denote a probability space and let $\mathscr{F}=\left(\mathcal{F}_{t}\right)_{t \in \mathbf{R}}$ be a filtration in $\mathcal{F}$. In the present paper we consider integrals of the form

$$
\int_{-\infty}^{\infty} \phi_{s} \cdot \mathrm{d} Z_{s}
$$

where $\phi$ is an $\mathbf{R}^{n}$-valued predictable process indexed by $\mathbf{R}$ and $Z=\left(Z_{t}\right)_{t \in \mathbf{R}}$ is an $\mathbf{R}^{n}$-valued increment semimartingale, i.e., a process for which $\left(Z_{s+t}-\right.$ $\left.Z_{s}\right)_{t \geqslant 0}$ is a semimartingale in the usual sense with respect to $\left(\mathcal{F}_{s+t}\right)_{t \geqslant 0}$ for all $s \in \mathbf{R}$. If $Z$ is a semimartingale, it is adapted and $Z_{-\infty}=\lim _{t \rightarrow-\infty} Z_{t}$ exists a.s. (See the next section for the definition of semimartingales indexed by $\mathbf{R}$.) However, when the index set is $\mathbf{R}$, there are important examples of increment semimartingales that are not semimartingales; indeed, if $Z$ is a Lévy process indexed by $\mathbf{R}$, the limit $Z_{-\infty}$ does not exist except in trivial cases. But in Subsection 4.2 we show that $Z$ is an increment semimartingale in the filtration $\mathcal{F}_{t}=\mathcal{F}_{t}^{Z}$, where $\mathcal{F}_{t}^{Z}=\sigma\left(Z_{s}: s \in(-\infty, t]\right)$. Hence, it is important to develop a theory of integration beyond semimartingales.

\footnotetext{
${ }^{*}$ Department of Mathematics, The University of Tennessee, USA; e-mail: aboc@math.utk.edu

${ }^{* *}$ Department of Mathematical Sciences, University of Aarhus, Denmark; e-mail: matseg@imf.au.dk; jan@imf.au.dk
} 
When the index set is $\mathbf{R}_{+}$rather than $\mathbf{R}, Z$ is a semimartingale and $t>0$, it is well known, cf., e.g., [17], how to characterize the set of predictable processes $\phi$ for which $\int_{0}^{t} \phi_{s} \cdot d Z_{s}$ exists in terms of the triplet of $Z$. Cherny and Shiryaev [11] have generalized this to integrals of the form $\int_{0}^{\infty} \phi_{s} \cdot \mathrm{d} Z_{s}$. Extending these results we shall in the present paper characterize the set $L(Z)$ of integrable processes in (1.1) in terms of the characteristic triplet of $Z$; see Theorem 3.2. In particular, we show that when $\phi \in L(Z)$ the process $\int_{-\infty}^{t} \phi_{s} \cdot \mathrm{d} Z_{s}, t \in \mathbf{R}$, is a semimartingale up to infinity. See also [4] for integration with respect to increment local martingales.

Important examples of (1.1) include

$$
(\alpha): \quad \int_{-\infty}^{\infty}\left(g(t-s) \sigma_{s}\right) \cdot \mathrm{d} Z_{s} \quad \text { and } \quad(\beta): \quad \int_{-\infty}^{\infty} f\left(s, Z_{s-}\right) \cdot \mathrm{d} Z_{s},
$$

where $Z$ is an $\mathbf{R}^{n}$-valued Lévy process indexed by $\mathbf{R}, \sigma$ is an $\mathbf{R}^{n}$-valued predictable stationary process, $g$ is an $\mathbf{R}^{n^{2}}$-valued deterministic function, $t \in$ $\mathbf{R}$, and $f: \mathbf{R} \times \mathbf{R}^{n} \rightarrow \mathbf{R}^{n}$ is a measurable function. The setting $(\alpha)$ is in [1][3] used for modeling of an interesting new class of moving averages; here we recall, e.g., from [13] that moving averages provide a large class of stationary processes. Note that when in $(\alpha) \sigma$ and $Z$ are independent it is easy to define the integral by conditioning on $\sigma$ and using integration of a deterministic integrand with respect to an independently scattered random measure, cf., e.g., [23]. However, beyond this case it seems to be necessary to use an approach as in the present paper. Integrals as in $(\beta)$ show up, for instance, in the study of stationary solutions of linear stochastic differential equations. As an example assume that $\left(Z_{t}\right)=\left(\eta_{t}, \xi_{t}\right)$ is a bivariate Lévy process indexed by $\mathbf{R}$ with $\left(\eta_{0}, \xi_{0}\right)=(0,0)$ such that $\xi_{t} \rightarrow \infty$ as $t \rightarrow \infty$. Let $\left(V_{t}\right)_{t \in \mathbf{R}}$ be a generalized Ornstein-Uhlenbeck process given by $V_{t}=e^{-\left(\xi_{t}-\xi_{s}\right)} V_{s}+$ $e^{-\xi_{t}} \int_{s}^{t} e^{\xi_{u}-} \mathrm{d} \eta_{u}$ for $s<t$. See [7] for more information about generalized Ornstein-Uhlenbeck processes and references to theory and applications of these. Using the results of the present paper, it was shown in [5] that in case of stationarity $V$ is given by

$$
V_{t}=e^{-\xi_{t}} \int_{-\infty}^{t} e^{\xi_{s-}} \mathrm{d} \eta_{s}, \quad t \in \mathbf{R}
$$

where the integral in (1.3), as well as $(\beta)$ in general, is defined in the filtration $\mathscr{F}^{Z}$. As mentioned above, $\eta$ is an increment semimartingale in $\mathscr{F}^{Z}$. Equation (1.3) generalizes in a natural way a well-known representation of ordinary Ornstein-Uhlenbeck processes which corresponds to $\xi_{t}=\lambda t$ for some $\lambda>0$.

As we already have argued a Lévy process $Z$ cannot be a semimartingale in any filtration. Further complications arise due to the following facts: (i) $Z$ is not an $\mathscr{F}^{Z}$-Lévy process (because $Z_{t}-Z_{s}$ is not independent of $\mathcal{F}_{s}^{Z}$ for all $-\infty<s<t<\infty$ ); (ii) even when $Z$ is centered it is not a martingale 
in $\mathcal{F}^{Z}$ (or in any other filtration). Thus, the above-mentioned result that $Z$ is an increment semimartingale in $\mathscr{F}^{Z}$ is not trivial. This result relies on an expansion of filtration result extending [16, Theorems 2.6 and 2.9]. In $\mathscr{F}^{Z}$ it does however not seem possible to calculate the triplet of $Z$ explicitly. Therefore, in Theorem 4.1, we consider an expanded filtration in which $Z$ still is an increment semimartingale and in which we are able to calculate the triplet of $Z$ explicitly; this gives, in particular, applicable conditions for integrals of the form $(\beta)$ to exist in the extended filtration as well as in $\mathscr{F}^{Z}$. Theorem 4.3 concerns the important case of a square integrable Lévy process.

Finally, let us briefly explain how we characterize the set of integrable processes $L(Z)$. First of all, if $Z$ is a semimartingale up to infinity, then it is well known from the Bichteler-Dellacherie theorem that $Z$ induces a finite $n$-dimensional $L^{0}$-valued vector measure defined as $m(A)=\int_{A} \mathrm{~d} Z_{s}$ for any predictable set $A$. More generally, we see in Example 3.1 that an increment semimartingale induces a so-called Radon measure, which is a particular example of a $\sigma$-finite measure on the predictable $\sigma$-field. Thus, generalizing (1.1), we consider also

$$
\int_{-\infty}^{\infty} \phi_{s} \cdot m(\mathrm{~d} s)
$$

where $m$ is any $\sigma$-finite vector measure on the predictable $\sigma$-field. The point is that there is a well-developed theory of integration with respect to vector measures, where the set of integrable processes is characterized in terms of the so-called semivariation of $m$. We apply this theory to characterize $L(m)$ and $L(Z)$. This approach turns out to be both natural, simple and powerful.

In the case when $Z=\left(Z_{s}\right)_{s \in[0, t]}$ is quasi-left continuous semimartingale and $n=1$, Kwapień and Woyczyński [18, Theorem 6.1] have characterized the topology on the set of integrable functions $L(Z)$ in terms of the triplet of $Z$. We extend in Theorem 3.3 this result to the case of integrals of the form (1.4), i.e., to $\sigma$-finite $L^{0}$-valued measures. To prove this we show and apply a characterization of convergence in Emery's semimartingale topology, see Theorem 3.5. The triplet of a $\sigma$-finite $L^{0}$-valued measure $m$ is introduced in Theorem 3.1.

Section 5 gives the necessary background on integration theory for $\sigma$ finite measures $m$ on a measurable space $(E, \mathscr{E})$ with values in a linear metric space $F$. In particular, we see how $m$ induces (in a canonical way) a set of integrable functions, called $L(m)$, by its semivariation. See, e.g., [8][10], [19], [24], [28] for nice treatments of integration with respect to vector valued measures. However, only few of these references consider $\sigma$-finite rather than finite measures; one notable exception is [28], where a $\sigma$-finite measure is called a formal measure. Our approach differs slightly from that of [28], e.g., we start with $\sigma$-additive set functions $m$ instead of integral 
mappings with certain continuity properties. In what follows focus is on the case $(E, \mathscr{E})=(\mathbf{R} \times \Omega, \mathscr{P})$ and $F=L^{0}$.

2. Notation and basic definitions. Throughout the rest of the paper $(\Omega, \mathcal{F}, \mathbf{P})$ denotes a probability space and $L^{0}$ is $L^{0}(\Omega, \mathcal{F}, \mathbf{P})$. Recall that with $\|Z\|_{0}=\mathbf{E}[|Z| \wedge 1]$ for $Z \in L^{0},\left(L^{0},\|\cdot\|_{0}\right)$ is an $F$-space, i.e., $L^{0}$ is a linear space and $\|\cdot\|_{0}$ induces a complete metric. See Section 5 for properties of $F$-spaces.

Let $\mathscr{F}=\left(\mathcal{F}_{t}\right)_{t \in \mathbf{R}}$ be a filtration, i.e., an increasing family of $\sigma$-fields satisfying the usual conditions of right-continuity and completeness. Set $\mathcal{F}_{-\infty}=\bigcap_{t \in \mathbf{R}} \mathcal{F}_{t}$ and $\mathcal{F}_{\infty}=\sigma\left(\bigcup_{t \in \mathbf{R}} \mathcal{F}_{t}\right)$. If $-\infty<a<b<\infty$, then as usual, an $\mathbf{R}^{n}$-valued process indexed by $[a, b]$ or $[a, \infty)$ is said to be a semimartingale with respect to $\left(\mathcal{F}_{t}\right)_{t \in[a, b]}$, respectively, $\left(\mathcal{F}_{t}\right)_{t \in[a, \infty)}$, if it can be decomposed into the sum of an $\mathbf{R}^{n}$-valued càdlàg local martingale with respect to the same filtration and an $\mathbf{R}^{n}$-valued càdlàg adapted process of bounded variation on compacts; see [17] for the basic properties. An $\mathbf{R}^{n}$-valued process $X=\left(X_{t}\right)_{t \in \mathbf{R}}$ is a semimartingale with respect to $\mathscr{F}$ if $X_{-\infty}=\lim _{t \rightarrow-\infty} X_{t}$ exist a.s. and for all continuous and increasing functions $g:[0, \infty) \rightarrow\left[-\infty, \infty\left[, \quad\left(X_{g(t)}\right)_{t \in[0, \infty)}\right.\right.$ is an $\left(\mathcal{F}_{g(t)}\right)_{t \in[0, \infty)}$-semimartingale. Finally, an $\mathbf{R}^{n}$-valued process $X=\left(X_{t}\right)_{t \in \mathbf{R}}$ is called a semimartingale up to infinity with respect to $\mathscr{F}$ if $X_{-\infty}=\lim _{t \rightarrow-\infty} X_{t}$ and $X_{\infty}=\lim _{t \rightarrow \infty} X_{t}$ exist a.s. and for all continuous and increasing functions $f:[0,1] \rightarrow[-\infty, \infty]$,

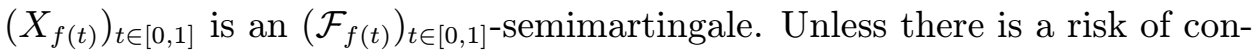
fusion the filtration will typically not be mentioned explicitly.

Let $\mathcal{S M}$ be the space of all real-valued semimartingales up to infinity $X=\left(X_{t}\right)_{t \in \mathbf{R}}$ equipped with Emery's [14] semimartingale topology

$$
\|X\|_{\mathcal{S M}}=\sup _{\phi \in M(\mathscr{P} ; \mathbf{R}),|\phi| \leqslant 1}\left\|\int_{-\infty}^{\infty} \phi_{s} \mathrm{~d} X_{s}\right\|_{0}, \quad X \in \mathcal{S M}
$$

where $\mathscr{P}$ is the predictable $\sigma$-field, see $(2.2)$ below. Recall that $\left(\mathcal{S M},\|\cdot\|_{\mathcal{S M}}\right)$ is an $F$-space (cf. [14] and [21]).

An $\mathbf{R}^{n}$-valued process $X=\left(X_{t}\right)_{t \in \mathbf{R}}$ is called an increment semimartingale if for all $a \in \mathbf{R}$ the process $\left(X_{t}-X_{a}\right)_{t \geqslant a}$ is a semimartingale. Note that an increment semimartingale need not be adapted but all increments are adapted. The class of increment local martingales, defined in the obvious way, is studied in [4]. In particular, [4, Remark 3.15] shows that if $X$ is a continuous increment local martingale such that $X_{-\infty}$ exist a.s., then the process $X-X_{-\infty}$ is a continuous local martingale. There is no such result for continuous increment semimartingales. For example, if $g$ : $\mathbf{R} \rightarrow \mathbf{R}$ is a continuous function for which $g_{-\infty}=\lim _{t \rightarrow-\infty} g_{t}$ exists and which is of bounded variation on compacts but of unbounded variation on $(-\infty, 0)$, then $\left(g_{t}\right)_{t \in \mathbf{R}}$ is an increment semimartingale but not a semimartingale. 
Whenever $X=\left(X_{t}\right)_{t \in \mathbf{R}}$ is a semimartingale and $\tau$ is a truncation function on $\mathbf{R}^{n}$, i.e., $\tau: \mathbf{R}^{n} \rightarrow \mathbf{R}^{n}$ is a bounded function such that $\tau(x)=x$ in a neighborhood of zero, define $X(\tau)$ as

$$
X_{t}(\tau)=X_{t}-\sum_{s \in(-\infty, t]}\left(\Delta X_{s}-\tau\left(\Delta X_{s}\right)\right), \quad t \in \mathbf{R} .
$$

Moreover, let $\mu^{X}$ denote the jump measure of $X$, that is, with $\mathbf{R}_{0}^{n}=\mathbf{R}^{n} \backslash\{0\}$,

$$
\mu^{X}(A)=\sharp\left\{s \in \mathbf{R}:\left(s, \Delta X_{s}\right) \in A\right\}, \quad A \in \mathscr{B}\left(\mathbf{R} \times \mathbf{R}_{0}^{n}\right) .
$$

Let $(U, \mathscr{U})$ be a measurable space. Then $\mu=\mu(\omega ; \mathrm{d} u)$ is said to be a random signed measure on $U$ if for all $\omega \in \Omega, \mu(\omega ; \cdot)$ is an $\mathbf{R}$-valued $\sigma$ finite measure on $(U, \mathscr{U})$ as defined in Subsection 5.1; i.e., $\mu(\omega ; \varnothing)=0$ and there exist $\left(A_{l}\right)_{l \geqslant 1} \subseteq \mathscr{U}$ (depending in general on $\omega$ ) such that $A_{l} \uparrow U$ and for all disjoint sets $\left(B_{k}\right)_{k \geqslant 1} \subseteq \mathscr{U}$ with $\bigcup_{k=1}^{\infty} B_{k} \subseteq A_{l}$ for some $l \geqslant 1$ we have $\mu\left(\omega ; \bigcup_{k=1}^{\infty} B_{k}\right)=\sum_{k=1}^{\infty} \mu\left(\omega ; B_{k}\right)$ in $\mathbf{R}$. In case $A_{l}=U$ for all $l, \mu$ is called a finite random signed measure on $U$. If for all $\omega, \mu(\omega ; \cdot)$ takes values in $[0, \infty), \mu$ is called a random positive measure. In this case $\mu(\omega ; \cdot)$ extends to a $[0, \infty]$-valued measure defined on the entire $\sigma$-field $\mathscr{U}$, and it is then a random measure in the sense of [17]. Denote by $\operatorname{Var}(\mu ; \cdot)$ the total variation of the random signed measure $\mu$, that is, the positive random measure, finite on each $A_{l}$, given, for $A \in \mathscr{U}$ with $A \subseteq A_{l}$ for some $l$, by

$$
\begin{array}{r}
\operatorname{Var}(\mu ; A)(\omega)=\sup \left\{\sum_{i=1}^{k}\left|\mu\left(\omega ; B_{i}\right)\right|: k \geqslant 1,\left(B_{i}\right)_{i=1}^{k} \subseteq \mathscr{U}\right. \\
\text { are disjoint with } \left.\bigcup_{i=1}^{k} B_{i}=A\right\} .
\end{array}
$$

When $U=\mathbf{R}^{n}$ and $\mu(\omega ;\{u\})=0$ for all $u \in U$ and $\omega \in \Omega$, we say that $\mu$ is continuous. When $\mu=\left(\mu^{i}\right)_{i \leqslant n}$, where each $\mu^{i}$ is a random signed measure on $U$, we speak of $\mu$ as an $n$-dimensional random signed measure on $U$.

Let $\mathscr{P}$ denote the predictable $\sigma$-field on $\mathbf{R} \times \Omega$, i.e.,

$$
\mathscr{P}=\sigma\left((u, t] \times A:-\infty<u<t<\infty, A \in \mathcal{F}_{u}\right),
$$

and let $\widetilde{P}=\mathscr{P} \otimes \mathscr{U}$ and $\widetilde{\Omega}=\mathbf{R} \times \Omega \times U$. A random measure $\mu$ on $\mathbf{R} \times U$ is said to be $\widetilde{\mathscr{P}}_{-} \sigma$-finite if there exists $\left(A_{l}\right)_{l \geqslant 1} \subseteq \widetilde{\mathscr{P}}$ such that $A_{l} \uparrow \widetilde{\Omega}$ and $\mathbf{E}\left[\int 1_{A_{l}}(s, x) \operatorname{Var}(\mu ; \mathrm{d} s \times \mathrm{d} x)\right]<\infty$ for all $l \geqslant 1$. For any $\widetilde{\mathscr{P}}_{\text {-measurable }}$ functions $W=W(s, \omega, x)$ we will use the standard notation

$$
W * \mu_{t}=\int_{-\infty}^{t} \int_{U} W(s, x) \mu(\mathrm{d} s \times \mathrm{d} x)
$$

and

$$
W * \mu=\int_{\mathbf{R}} \int_{U} W(s, x) \mu(\mathrm{d} s \times \mathrm{d} x),
$$


whenever the integrals are well defined. Furthermore, $\mu$ is called predictable if for all $\widetilde{P}$-measurable functions $W,\left(W * \mu_{t}\right)_{t \in \mathbf{R}}$ is predictable whenever it is well defined. Optional random signed measures are defined similarly, see [17, Chap. II, Section 1]. Finally, a random signed measure $\mu$ on $\mathbf{R}$

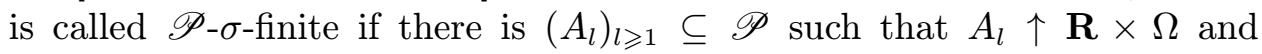
$\mathbf{E}\left[\int 1_{A_{l}}(s) \operatorname{Var}(\mu ; \mathrm{d} s)\right]<\infty$ for all $l \geqslant 1$.

3. Integration of predictable processes. In this section we consider the stochastic integral on the predictable $\sigma$-field with respect to a $\sigma$-finite $L^{0}$-valued measure (cf. Section 5). In [28] such measures are called formal semimartingales; see also [15] and [10]. A key point in this paper is the introduction of the characteristic triplet of $\sigma$-finite measures; see Theorem 3.1. In the case of a finite measure (which corresponds to a semimartingale) this notion has been very successfully applied, e.g., in [17]. Thereafter, in Theorems 3.2 and $3.3, L(m)$ is characterized in terms of the triplet of $m$, giving applicable conditions for a process to be integrable.

Consider the setting of Section 5 in the case, where $(E, \mathscr{E})=(\mathbf{R} \times \Omega, \mathscr{P})$; thus let $m=\left(m^{i}\right)_{i \leqslant n}$ denote an $n$-dimensional $\sigma$-finite $L^{0}$-valued measure on $(\mathbf{R} \times \Omega, \mathscr{P})$ and let $\left(O_{k}\right)_{k \geqslant 1} \subseteq \mathscr{P}$ satisfying $O_{k} \uparrow \mathbf{R} \times \Omega$ be $m^{i}$-feasible for all $i$; that is, $m$ is defined on $\mathscr{P}_{m}=\left\{A \in \mathscr{P}: A \subseteq O_{k}\right.$ for some $\left.k \geqslant 1\right\}$ and for any disjoint sequence $\left(A_{k}\right)_{k \geqslant 1} \subseteq \mathscr{P}_{m}$ with $\bigcup_{k=1}^{\infty} A_{k} \in \mathscr{P}_{m}$ we have $m\left(\bigcup_{k=1}^{\infty} A_{k}\right)=\sum_{k=1}^{\infty} m\left(A_{k}\right)$ in $L^{0}$. For all $t \in \mathbf{R}$ let $\Omega_{t}=(-\infty, t] \times \Omega$ and assume throughout that

(1) $\forall A \in \mathscr{P}_{m}, A \subseteq \Omega_{t}: m(A)$ is $\mathcal{F}_{t}$-measurable,

(2) $\forall A \in \mathscr{P}_{m}, u \in \mathbf{R}, B \in \mathcal{F}_{u}: m(A \cap((u, \infty) \times B))=1_{B} m(A \cap$ $((u, \infty) \times \Omega))$.

Recall from Section 5 that $m$ is called a finite measure if one can take $O_{k}=\mathscr{P}$, i.e., $m$ can be defined on the entire predictable $\sigma$-field.

$\mathrm{Ex}$ a $\mathrm{m} \mathrm{ple} \mathrm{3.1.} \mathrm{If} m$ is a finite measure, then the $\mathbf{R}^{n}$-valued process $X=\left(X_{t}\right)_{t \in \mathbf{R}}$ defined by $X_{t}=m\left(\Omega_{t}\right)$ can be chosen càdlàg and is then an $\left(\mathcal{F}_{t}\right)_{t \in \mathbf{R}}$-semimartingale up to infinity with $X_{-\infty}=0$ by the Bichteler-Dellacherie theorem (see [9] or [28]). Conversely, if we start out

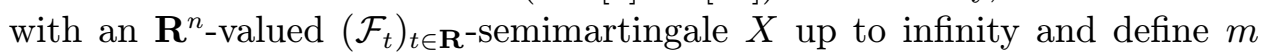
by $m(A)=\int_{A} 1 \mathrm{~d} X$ for $A \in \mathscr{P}$ (where the integral is defined coordinatewise in the semimartingale sense), then $m$ is a finite measure. When $m$ is a finite measure we will often not distinguish between $m$ and the corresponding semimartingale. In this case the integral $\int \phi \cdot \mathrm{d} m$ is well defined and given as $\int \phi \cdot \mathrm{d} m=\sum_{i=1}^{n} \int \phi^{i} \mathrm{~d} m^{i}$ whenever $\phi=\left(\phi^{i}\right)_{i \leqslant n}$ is an $\mathbf{R}^{n}$-valued bounded predictable process.

Another important example is when $m$ is a Radon measure, by which we mean that it is a $\sigma$-finite $L^{0}$-valued measure with $O_{k}=[-k, k] \times \Omega$, $k \geqslant 1$. As a consequence of the Bichteler-Dellacherie theorem it follows that $m$ is a Radon measure if and only if there exists an $\mathbf{R}^{n}$-valued increment 
semimartingale $Z=\left(Z_{t}\right)_{t \in \mathbf{R}}$ with respect to $\mathscr{F}$ such that $m((u, t] \times B)=$ $1_{B}\left(Z_{t}-Z_{u}\right)$ for all $-\infty<u<t<\infty$ and $B \in \mathcal{F}_{u}$.

Recall from Section 5 that an $\mathbf{R}^{n}$-valued predictable process $\phi=\left(\phi^{i}\right)_{i \leqslant n}$ induces a one-dimensional $\sigma$-finite $L^{0}$-valued measure on $(\mathbf{R} \times \Omega, \mathscr{P})$ defined as $\phi \bullet m(A)=\int_{A} \phi \cdot \mathrm{d} m$ for any predictable set $A$ satisfying $A \subseteq \bar{O}_{k}=O_{k} \cap$ $\{\|\phi\| \leqslant k\}$ for some $k$. Note that the integral is well defined, since $m\left(\cdot \cap O_{k}\right)$ is a finite measure and $1_{A} \phi$ is a bounded predictable process. Moreover, $\phi \bullet m$ satisfies (1) and (2). The set of integrable predictable processes $\phi$ with respect to $m$ (in the sense of Section 5 ) is denoted by $L(m)$, and $L^{1}(m)$ is the subset of $L(m)$ for which $\int \phi \cdot \mathrm{d} m$ can be defined as $\int \phi \cdot \mathrm{d} m=\sum_{i=1}^{n} \int \phi^{i} \mathrm{~d} m^{i}$. When $m$ is induced by an increment semimartingale $Z$ we often write $L(Z)$ instead of $L(m)$ and $\int \phi \cdot \mathrm{d} Z$ instead of $\int \phi \cdot \mathrm{d} m$. In the following we shall use both the characterization of $L(m)$ given in (5.3) as well as the fact that $\phi \in L(m)$ if and only if $\phi \bullet m$ induces a finite one-dimensional $L^{0}$-valued measure corresponding to a semimartingale up to infinity as discussed in Example 3.1. In this case $\int \phi \cdot \mathrm{d} m$ is defined as $\phi \bullet m(\mathbf{R} \times \Omega)$. Some of the basic properties of the integral are summarized in Theorem 5.2. According to (5.2) and Theorem 5.2 the semivariation $\|\phi\|_{m}$ is

$$
\|\phi\|_{m}=\sup \left\{\left\|\int \psi \mathrm{d}(\phi \bullet m)\right\|_{0}: \psi \in M(\mathscr{P} ; \mathbf{R}),|\psi| \leqslant 1\right\}, \quad \phi \in L(m),
$$

which by (2.1) shows that $\|\phi\|_{m}=\|\phi \bullet m\|_{\mathcal{S M}}$. This implies that $\left(L(m),\|\cdot\|_{m}\right)$ is an $F$-space. To see this we only have to argue that $\|\cdot\|_{m}$ induces a complete metric, and by using [28, p. 427, 428], we may and do assume that $m$ is a finite measure, that is, corresponds to an $\mathbf{R}^{n}$-valued semimartingale up to infinity. Since $\|\phi\|_{m}=\|\phi \bullet m\|_{\mathcal{S M}}$, the metric induced by $\|\cdot\|_{m}$ coincides with the one imposed by Mémin [21] so the result is given in his Theorem V.4.

For all $A \in \mathscr{P}$ and $\phi \in M\left(\mathscr{P} ; \mathbf{R}^{n}\right)$ satisfying $1_{A} \phi \in L(m)$ we use the notation $\int_{A} \phi \cdot \mathrm{d} m$ rather than $\int\left(1_{A} \phi\right) \cdot \mathrm{d} m$; if $A=(s, t] \times \Omega$ with $s<t$, we write $\int_{(s, t]}$ or $\int_{s}^{t}$ instead of $\int_{A}$ and similarly when $A=(s, \infty) \times \Omega$.

In a sense one can think of $\int \phi \cdot \mathrm{d} m$ as a proper integral. An alternative would be to consider improper integrals defined as $\lim _{t \rightarrow-\infty} \int_{t}^{\infty} \phi \cdot \mathrm{d} m$ provided this limit exists a.s. and $\phi 1_{\Omega_{t}^{c}} \in L(m)$ for all $t \in \mathbf{R}$. However, as is already known from ordinary Lebesgue integrals it is hard to give appropriate conditions for improper integrals to exist. Generally, if the proper integral exists, then so does the improper integral but the converse is only true in special cases. For example, let $\left(\xi_{t}, \eta_{t}\right)_{t \in \mathbf{R}}$ be a bivariate Lévy process indexed by $\mathbf{R}$ with $\left(\xi_{0}, \eta_{0}\right)=(0,0)$ and let $t>0$. Then the improper integral $\lim _{s \rightarrow-\infty} \int_{s}^{t} e^{\xi_{u}} \mathrm{~d} \eta_{u}$ exists if and only if $\left(1_{\{u \leqslant t\}} e^{\xi_{u}}\right)_{u \in \mathbf{R}} \in L(\eta)$; see [5]. The appropriate choice of filtration when working with Lévy processes is discussed in Section 4. In another direction, if $Z$ is a continuous increment local martingale, then improper and proper integrals at $-\infty$ exist at the same time; see [4]. 
3.1. The triplet of random measures. For all $k \geqslant 1$ let $X(k)=$ $\left\{\left(X_{t}^{i}(k)\right)_{i \leqslant n}: t \in \mathbf{R}\right\}$ be given by $X_{t}(k)=m\left(O_{k} \cap \Omega_{t}\right)$. As mentioned in Example 3.1 we may and will assume that $X(k)$ is a càdlàg $\mathscr{F}$-semimartingale up to infinity for all $k \geqslant 1$. Note that $X(k)$ satisfies

$$
X_{t}(k)=\int_{-\infty}^{t} 1_{O_{k}}(s) \mathrm{d} X_{s}(k+1), \quad k \geqslant 1, \quad t \in \mathbf{R} .
$$

Thus, if there exists a $k \geqslant 1$ (depending on $\omega$ ) such that $\Delta X_{t}^{i}(k)(\omega) \neq 0$, then $\Delta X_{t}^{i}(k)(\omega)=\Delta X_{t}^{i}(l)(\omega)$ for all $l>k$ and $i=1, \ldots, n$. Define $m$ 's jump process $\Delta m=\left\{\left(\Delta m_{t}^{i}\right)_{i \leqslant n}: t \in \mathbf{R}\right\}$ as

$$
\Delta m_{t}^{i}= \begin{cases}\Delta X_{t}^{i}(k) & \text { whenever } \Delta X_{t}^{i}(k) \neq 0 \text { for some } k \geqslant 1 \\ 0 & \text { otherwise }\end{cases}
$$

and $m$ 's jump measure $\mu$ as

$$
\mu(A)=\sharp\left\{t \in \mathbf{R}:\left(t, \Delta m_{t}\right) \in A\right\}, \quad A \in \mathscr{B}\left(\mathbf{R} \times \mathbf{R}_{0}^{n}\right) .
$$

For every $A \in \mathscr{P}$ such that $1_{A} \in L^{1}\left(m^{i}\right)$ for $i=1, \ldots, n$, define the semimartingale up to infinity $\left(m(A)_{t}\right)_{t \in \mathbf{R}}$ as $m(A)_{t}=\left(m^{i}(A)_{t}\right)_{i \leqslant n}=m\left(A \cap \Omega_{t}\right)$. Call $m$ a continuous martingale measure if for all such $A$ the process $\left(m(A)_{t}\right)_{t \in \mathbf{R}}$ is an $n$-dimensional continuous local martingale. Note that since each $X(k)$ is a semimartingale, its jump measure is finite. Consequently, $\mu$, the jump measure of $m$, is a $\widetilde{\mathscr{P}}_{-} \sigma$-finite integer-valued random measure on $\mathbf{R} \times \mathbf{R}_{0}^{n}$ and hence by [17, Theorem II.1.8] has a predictable compensator $\nu$. Thus, in particular $\nu$ is a $\widetilde{\mathscr{P}}_{-} \sigma$-finite measure on $\mathbf{R} \times \mathbf{R}_{0}^{n}$. Denote by $G_{\text {loc, } \infty}(\mu)$ the set of $\mathbf{R}$-valued $\widetilde{P}$-measurable functions $W=W(s, \omega, x)$ for which $W *(\mu-\nu)$ exists up to infinity. That is, there is a localizing sequence $\left(\sigma_{k}\right)_{k \geqslant 1}$ with $\mathbf{P}\left(\sigma_{k}=\infty\right) \rightarrow 1$ such that, with $\widetilde{W}$ defined as in [17, Definition II.1.27] we have $\mathbf{E}\left[\left(\sum_{s \leqslant \sigma_{k}}\left(\widetilde{W}_{s}\right)^{2}\right)^{1 / 2}\right]<\infty$. We use the notation $W *(\mu-\nu)$ for the integral over $\mathbf{R} \times \mathbf{R}_{0}^{n}$ of $W$ with respect to $\mu-\nu$.

The following result concerns the canonical decomposition of $m$ relative to a truncation function $\tau$. Other decomposition results of $L^{p}$-valued measure are studied in [10]. As usual we generally suppress $\omega$ in random variables.

Theorem 3.1. There exist a continuous martingale measure $m^{c}=$ $\left(m^{c, i}\right)_{i \leqslant n}$, a predictable compensator $\nu$ of $\mu$, and an $n$-dimensional $\mathscr{P}-\sigma$ finite random signed measure $B=\left(B^{i}\right)_{i \leqslant n}$ on $\mathbf{R}$ such that for all $A \in \mathscr{P}_{m}$, $1_{A} \in L^{1}\left(m^{c, i}\right)$ for $i=1, \ldots, n$, and

$$
m(A)=m^{c}(A)+\left(1_{A}(s) \tau(x)\right) *(\mu-\nu)+\left(1_{A}(s)(x-\tau(x))\right) * \mu+\int_{\mathbf{R}} 1_{A}(s) B(\mathrm{~d} s) .
$$


Furthermore, there exists an $(n \times n)$-dimensional predictable $\mathscr{P}$ - $\sigma$-finite random signed measure $C=\left(C^{i, j}\right)_{i, j \leqslant n}$ on $\mathbf{R}$ such that for $i, j=1, \ldots, n$ and $t \in \mathbf{R}$

$$
\left\langle m^{c, i}(A) ., m^{c, j}(A) .\right\rangle_{t}=\int_{-\infty}^{t} 1_{A}(s) C^{i, j}(\mathrm{~d} s), \quad A \in \mathscr{P}_{m},
$$

where $\langle\cdot, \cdot\rangle_{t}$ denotes the predictable quadratic variation. Moreover, $(B, C, \nu)$ is unique.

Finally, there are a $\mathscr{P}$ - $\sigma$-finite predictable random positive measure $\lambda=\lambda(\omega ; \mathrm{d} s)$ on $\mathbf{R}$, two predictable processes, $b=\left\{\left(b_{t}^{i}\right)_{i \leqslant n}: t \in \mathbf{R}\right\}$ with values in $\mathbf{R}^{n}$ and $c=\left\{\left(c_{t}^{i, j}\right)_{i, j \leqslant n}: t \in \mathbf{R}\right\}$ taking values in the symmetric positive semidefinite $(n \times n)$-matrices, and a transition kernel $K=$ $K(s, \omega ; \mathrm{d} x)$ from $(\mathbf{R} \times \Omega, \mathscr{P})$ into $\left(\mathbf{R}^{n}, \mathscr{B}\left(\mathbf{R}^{n}\right)\right)$ such that $K(s ;\{0\})=0$ and $\int_{\mathbf{R}^{n}}\left(1 \wedge\|x\|^{2}\right) K(s ; \mathrm{d} x)<\infty$ for all $s \in \mathbf{R}$,

$$
B(\mathrm{~d} s)=b_{s} \lambda(\mathrm{d} s), \quad C(\mathrm{~d} s)=c_{s} \lambda(\mathrm{d} s), \quad \nu(\mathrm{d} s \times \mathrm{d} x)=K(s ; \mathrm{d} x) \lambda(\mathrm{d} s) .
$$

The triplet $(B, C, \nu)$ given in Theorem 3.1 is called the triplet of $m$ and will play an important role in this paper. From the proof follows that if $m$ is a finite measure, then so is $m^{c}$. When $Z=\left(Z_{t}\right)_{t \in \mathbf{R}}$ is an increment semimartingale with associated random measure $m,(B, C, \nu)$ will also be called the triplet of $Z$.

$\mathrm{R}$ e $\mathrm{m}$ a r k 3.1. (1) Let us describe the right-hand side of (3.1) in more detail. The second term is defined coordinatewise and $W(s, x)=$ $1_{A}(s) \tau^{i}(x)$ is in $G_{\mathrm{loc}, \infty}(\mu)$ for all $i=1, \ldots, n$ ( $\tau^{i}$ is the $i$ th coordinate). The third term is defined coordinatewise as well and is in fact just a finite sum. Finally, $\int 1_{A}(s) \operatorname{Var}\left(B^{i} ; \mathrm{d} s\right)<\infty$ a.s. for all $i=1, \ldots, n$.

(2) In Section 5 it is shown that $L(m)$ is invariant under the choice of $\left(O_{k}\right)_{k \geqslant 1}$. Similarly, it follows by uniqueness that the triplet is invariant under the choice of $\left(O_{k}\right)_{k \geqslant 1}$.

$\mathrm{P}$ r o of of $\mathrm{T} \mathrm{h}$ e or e $\mathrm{m} 3.1$. For $k \geqslant 1$ let $m_{k}$ be the finite measure defined by $m_{k}(A)=m\left(A \cap O_{k}\right)$ for $A \in \mathscr{P}$. In other words, $m_{k}$ is the measure associated with $X(k)$ as described in Example 3.1. As shown in [17, Theorem II.2.34], there exists a unique triplet $\left(B_{k}, C_{k}, \nu_{k}\right)$ such that

$$
X_{t}(k)=X_{t}^{c}(k)+\tau(x) *\left(\mu^{X(k)}-\nu_{k}\right)_{t}+(x-\tau(x)) * \mu_{t}^{X(k)}+B_{k}((-\infty, t]),
$$

and so, denoting by $m_{k}^{c}$ the $n$-dimensional finite measure corresponding to $X^{c}(k)$, we get, for $A \in \mathscr{P}$,

$$
\begin{aligned}
m_{k}(A)= & m_{k}^{c}(A)+\left(1_{A}(s) \tau(x)\right) *\left(\mu_{k}-\nu_{k}\right)+\left(1_{A}(s)(x-\tau(x))\right) * \mu_{k} \\
& +\int_{\mathbf{R}} 1_{A}(s) B_{k}(\mathrm{~d} s) .
\end{aligned}
$$


Since

$$
m_{k}(A)=m_{k+1}(A) \text { for } A \in \mathscr{D}_{k}:=\left\{B \in \mathscr{P}: B \subseteq O_{k}\right\},
$$

we have $m_{k}^{c}=m_{k+1}^{c}$ on $\mathscr{D}_{k}$ by uniqueness. Hence we can define a $\sigma$-finite measure $m^{c}$ on $\mathscr{P}_{m}=\bigcup_{k=1}^{\infty} \mathscr{D}_{k}$ to be equal to $m_{k}^{c}$ on each $\mathscr{D}_{k}$. Again by (3.3) it follows that

$$
\begin{gathered}
\mu_{k}(\mathrm{~d} s \times \mathrm{d} x)=1_{O_{k}}(s) \mu_{k+1}(\mathrm{~d} s \times \mathrm{d} x), \\
B_{k}(\mathrm{~d} s)=1_{O_{k}}(s) B_{k+1}(\mathrm{~d} s), \quad C_{k}(\mathrm{~d} s)=1_{O_{k}}(s) C_{k+1}(\mathrm{~d} s),
\end{gathered}
$$

and therefore we may define $B(\mathrm{~d} s)$ to be equal to $B_{k}(\mathrm{~d} s)$ on $\mathscr{D}_{k}$ and $C(\mathrm{~d} s)$ to be equal to $C_{k}(\mathrm{~d} s)$ on $\mathscr{D}_{k}$. By construction of $(B, C, \nu),(3.1)$ holds. Note also that $\mu$ by definition equals $\mu_{k}$ on $\mathscr{D}_{k}$ for all $k \geqslant 1$ and hence $\nu=\sup _{k \geqslant 1} \nu_{k}$ by monotone convergence. The uniqueness of $(B, C, \nu)$ follows by the uniqueness of $\left(B_{k}, C_{k}, \nu_{k}\right)$ for all $k \geqslant 1$.

Due to the fact that $\nu$ is a $\widetilde{P}$ - $\sigma$-finite measure the existence of a $\mathscr{P}$ - $\sigma$-finite measure $\tilde{\lambda}$ and a transition kernel $\widetilde{K}$ such that $\nu(\mathrm{d} s \times \mathrm{d} x)=$ $\widetilde{K}(s ; \mathrm{d} x) \tilde{\lambda}(\mathrm{d} s)$ follows by general disintegration theory, see, e.g., $[17$, Chap. II, 1.2]. The construction of $b, c, K$, and $\lambda$ satisfying (3.2) is now obvious.

Throughout let $\tau_{1}$ and $\tau_{n}$ denote truncation functions on, respectively, $\mathbf{R}$ and $\mathbf{R}^{n}$.

Proposition 3.1. For all $\mathbf{R}^{n}$-valued predictable processes $\phi$, the $\sigma$ finite measure $\phi \bullet m$ has jump measure $\mu_{\phi \bullet m}$ and continuous martingale measure $(\phi \bullet m)^{c}$ given by

$$
\mu_{\phi \bullet m}=\mu \circ\left((s, x) \mapsto\left(s,\left\langle\phi_{s}, x\right\rangle\right)\right)^{-1}, \quad(\phi \bullet m)^{c}=\phi \bullet m^{c},
$$

and its triplet $(\widetilde{B}, \widetilde{C}, \widetilde{\nu})$ is given by

$$
\begin{gathered}
\widetilde{B}(\mathrm{~d} s)=\left(\left\langle\phi_{s}, b_{s}\right\rangle+\int_{\mathbf{R}^{n}}\left[\tau_{1}\left(\left\langle\phi_{s}, x\right\rangle\right)-\left\langle\phi_{s}, \tau_{n}(x)\right\rangle\right] K(s, \mathrm{~d} x)\right) \lambda(\mathrm{d} s), \\
\widetilde{C}(\mathrm{~d} s)=\left\langle\phi_{s}, c_{s} \phi_{s}\right\rangle \lambda(\mathrm{d} s), \quad \widetilde{\nu}=\nu \circ\left((s, x) \mapsto\left(s,\left\langle\phi_{s}, x\right\rangle\right)\right)^{-1} .
\end{gathered}
$$

Here for all $x, y \in \mathbf{R}^{n},\langle x, y\rangle=\sum_{i=1}^{n} x^{i} y^{i}$ denotes the usual inner product in $\mathbf{R}^{n}$. To prove Proposition 3.1 it is enough to consider each of the measures $m\left(\cdot \cap \bar{O}_{k}\right)$ for $k \geqslant 1$, where $\bar{O}_{k}$ is defined just below Example 3.1. However, $m\left(\cdot \cap \bar{O}_{k}\right)$ corresponds to an $\mathbf{R}^{n}$-valued semimartingale up to infinity and $\phi 1 \bar{O}_{k}$ is a bounded predictable process, so the results follows from [17, Chap. IX, Proposition 5.3].

Let $\phi \in L(m)$. Since in this case $\phi \bullet m$ is a finite measure, it follows from Theorem 3.1 and Proposition 3.1 that $\phi \in L\left(m^{c}\right), \tau_{1}\left(\left\langle\phi_{s}, x\right\rangle\right) \in G_{\mathrm{loc}, \infty}(\mu), \widetilde{B}$ 
is a finite random signed measure on $\mathbf{R}$ and

$$
\begin{aligned}
\int \phi \cdot \mathrm{d} m=\int \phi \cdot \mathrm{d} m^{c}+\tau_{1}( & \left(\left\langle\phi_{s}, x\right\rangle\right) *(\mu-\nu)+\left(\left\langle\phi_{s}, x\right\rangle\right. \\
& \left.-\tau_{1}\left(\left\langle\phi_{s}, x\right\rangle\right)\right) * \mu+\widetilde{B}((-\infty, \infty)) .
\end{aligned}
$$

3.2. A characterization of $L(m)$. In Section $5, L(m)$ is characterized in terms of the semivariation $\|\cdot\|_{m}$ which, however, is rarely known explicitly. The following result characterizes $L(m)$ in terms of the triplet $(B, C, \nu)$ of $m$ which is often known; see, e.g., Section 4 . We assume throughout this subsection that $m$ has triplet of the form (3.2) with respect to $\tau=\tau_{n}$.

Theorem 3.2. For any $\mathbf{R}^{n}$-valued predictable processes $\phi$ we have $\phi \in$ $L(m)$ if and only if the following (a)-(c) are satisfied almost surely:

$$
\begin{aligned}
& \text { (a) } \int_{\mathbf{R}}\left|\left\langle\phi_{s}, b_{s}\right\rangle+\int_{\mathbf{R}^{n}}\left[\tau_{1}\left(\left\langle\phi_{s}, x\right\rangle\right)-\left\langle\phi_{s}, \tau_{n}(x)\right\rangle\right] K(s ; \mathrm{d} x)\right| \lambda(\mathrm{d} s)<\infty, \\
& \text { (b) } \int_{\mathbf{R}}\left\langle\phi_{s}, c_{s} \phi_{s}\right\rangle \lambda(\mathrm{d} s)<\infty, \\
& \text { (c) } \int_{\mathbf{R}} \int_{\mathbf{R}^{n}}\left(1 \wedge\left|\left\langle\phi_{s}, x\right\rangle\right|^{2}\right) K(s ; \mathrm{d} x) \lambda(\mathrm{d} s)<\infty .
\end{aligned}
$$

Note that when $m$ is a continuous measure (i.e., $\mu=0)$, (3.5) and (3.6) reduce to

$$
\int_{\mathbf{R}}\left(\left|\left\langle\phi_{s}, b_{s}\right\rangle\right|+\left\langle\phi_{s}, c_{s} \phi_{s}\right\rangle\right) \lambda(\mathrm{d} s)<\infty \quad \text { a.s. }
$$

For integration with respect to increment local martingales see [4] and [6].

Set

$$
\begin{aligned}
& U(s, x)=\left|\left\langle x, b_{s}\right\rangle+\int_{\mathbf{R}^{n}}\left[\tau_{1}(\langle x, y\rangle)-\left\langle x, \tau_{n}(y)\right\rangle\right] K(s ; \mathrm{d} y)\right|, \\
& s \in \mathbf{R}, \quad x \in \mathbf{R}^{n}, \\
& \Phi(s, x)=\left\langle\begin{array}{c}
\left\langle x, c_{s} x\right\rangle+U(s, x)+\int_{\mathbf{R}^{n}}\left(1 \wedge|\langle x, y\rangle|^{2}\right) K(s ; \mathrm{d} y), \\
s \in \mathbf{R}, \quad x \in \mathbf{R}^{n},
\end{array}\right. \\
& L^{\Phi, 0}=\left\{\begin{array}{l}
\left.\phi \in M\left(\mathscr{P} ; \mathbf{R}^{n}\right): \int_{\mathbf{R}} \Phi\left(s, \phi_{s}\right) \lambda(\mathrm{d} s)<\infty \text { a.s. }\right\}, \\
\Psi_{\Phi, 0}(\phi)=\mathbf{E}\left[\left|\int_{\mathbf{R}} \Phi\left(s, \phi_{s}\right) \lambda(\mathrm{d} s)\right| \wedge 1\right], \quad \phi \in L^{\Phi, 0},
\end{array}\right.
\end{aligned}
$$

and $\widetilde{U}(s, x)=\sup _{c \in[-1,1]} U(s, c x)$. Recall that $A \in \mathscr{P}_{m}$ is called a null-set for $m$ if for all $B \in \mathscr{P}_{m}$ with $B \subseteq A, m(B)=0$. Moreover, a deterministic positive $\sigma$-finite measure $\kappa$ on $\mathscr{P}$ with the same null-sets as $m$ is called a control measure for $m$. The following gives more information about $L(m)$. 
Theorem 3.3. $L(m)=L^{\Phi, 0}$ and for all $\left(\phi^{k}\right)_{k \geqslant 1}$ and $\phi$ in $L(m), \phi^{k} \rightarrow$ $\phi$ in $L(m)$ if and only if $\Psi_{\Phi, 0}\left(\phi^{k}-\phi\right) \rightarrow 0$.

Moreover, $\lambda \otimes \mathbf{P}$, given by $(\lambda \otimes \mathbf{P})(A)=\mathbf{E}\left[\int_{\mathbf{R}} 1_{A}(s) \lambda(\mathrm{d} s)\right]$, is a control measure for $m$ whenever $\lambda$ is chosen such that $\lambda\left(s \in \mathbf{R}: b_{s}=0\right.$, $\left.c_{s}=0, K\left(s ; \mathbf{R}^{n}\right)=0\right)=0$ a.s.

When $n=1$ and $m$ is finite and quasi-left continuous, Theorem 3.3 is obtained in [18, Theorem 6.1] via decoupling techniques, whereas our approach is based on martingale theory.

Assume $n=1$ and set

$$
\begin{aligned}
\widetilde{\Phi}(s, x) & =c_{s} x^{2}+\widetilde{U}(s, x)+\int_{\mathbf{R}}\left(1 \wedge|x y|^{2}\right) K(s ; \mathrm{d} y), \\
\Psi_{\widetilde{\Phi}, 0}(\phi) & =\mathbf{E}\left[\left|\int_{\mathbf{R}} \widetilde{\Phi}\left(s, \phi_{s}\right) \lambda(\mathrm{d} s)\right| \wedge 1\right], \quad \phi \in L^{\Phi, 0} .
\end{aligned}
$$

From Musielak-Orlicz theory (see [22, Definition 7.2]) it follows that $\Psi_{\tilde{\Phi}, 0}$ is a modular. Moreover, by arguing as in [23] it can be shown that $\Psi_{\tilde{\Phi}, 0}$ satisfies the $\Delta_{2}$-condition, i.e., there exists a constant $v>0$ such that $\Psi_{\tilde{\Phi}, 0}(2 \phi) \leqslant$ $v \Psi_{\tilde{\Phi}, 0}(\phi)$ for all $\phi \in L^{\Phi, 0}$. Hence by [22, Theorems 1.5 and 7.7], $L^{\Phi, 0}$ is an $F$-space in the $F$-norm,

$$
\|\phi\|_{\tilde{\Phi}, 0}=\inf \left\{t>0: \Psi_{\tilde{\Phi}, 0}\left(\frac{\phi}{t}\right) \leqslant t\right\}
$$

and $\Psi_{\tilde{\Phi}, 0}\left(\phi^{k}\right) \rightarrow 0$ if and only if $\left\|\phi^{k}\right\|_{\tilde{\Phi}, 0} \rightarrow 0$. Therefore, by Lemma 3.2 below, we can restate Theorem 3.3 as follows.

Theorem 3.4. For $n=1, L(m)$ and $L^{\Phi, 0}$ are equivalent $F$-spaces, that is, the F-norms induce the same topology.

3.3. Proofs of Theorems 3.2, 3.3 and convergence in $\mathcal{S M}$. To prove Theorem 3.2 we need the following two lemmas.

Lemma 3.1. Let $\mu$ be a $\widetilde{\mathscr{P}}_{-} \sigma$-finite optional random positive measure on $\mathbf{R}_{0}^{n}$ with predictable compensator $\nu$ and let $W=W(s, \omega, x)$ be a positive

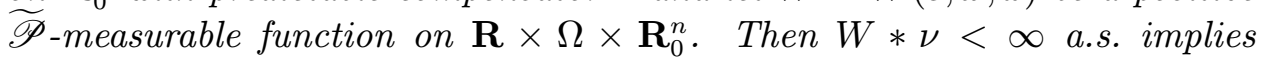
$W * \mu<\infty$ a.s.

P r o o f. For all $k \geqslant 1$ let $\sigma_{k}=\inf \left\{t \in \mathbf{R}: W * \nu_{t}>k\right\}$ which is a predictable stopping time, since $\left(W * \nu_{t}\right)_{t \in \mathbf{R}}$ is a predictable process, and hence $\left(-\infty, \sigma_{k}\right) \in \mathscr{P}$ for all $k \geqslant 1$. Therefore,

$$
\mathbf{E}\left[\left(W 1_{\left(-\infty, \sigma_{k}\right)}\right) * \mu\right]=\mathbf{E}\left[\left(W 1_{\left(-\infty, \sigma_{k}\right)}\right) * \nu\right] \leqslant k,
$$

implying $W * \mu_{\sigma_{k}-}<\infty$ a.s. for all $k \geqslant 1$. Furthermore, since $\sigma_{k}=\infty$ for $k$ sufficiently large we obtain $W * \mu<\infty$ a.s.

The next lemma follows as Lemma 2.8 in [23]. 
Lemma 3.2. There exists a constant $C_{1} \in(0, \infty)$, only depending on $\tau_{1}$, such that

$$
\widetilde{U}(s, x) \leqslant U(s, x)+C_{1} \int_{\mathbf{R}^{n}}\left(1 \wedge|\langle x, y\rangle|^{2}\right) K(s ; \mathrm{d} y), \quad s \in \mathbf{R}, \quad x \in \mathbf{R}^{n} .
$$

P r o o f of $\mathrm{Th}$ e o r e $\mathrm{m} 3.2$. Let $\phi \in L(m)$. In this case $\phi \bullet m$ is a finite measure, and its triplet $(\widetilde{B}, \widetilde{C}, \widetilde{\nu})$ is given in Proposition 3.1. From usual semimartingale theory we know that $\widetilde{B}$ has finite total variation on $(-\infty, \infty)$ (implying (a)), $\widetilde{C}$ has finite total variation on $(-\infty, \infty)$ a.s. (implying (b)) and finally $\int_{\mathbf{R} \times \mathbf{R}}\left(1 \wedge|y|^{2}\right) \widetilde{\nu}(\mathrm{d} s \times \mathrm{d} y)<\infty$ a.s. (implying (c)).

Conversely, assume that $\phi$ satisfies (3.5) and (3.6) and let us show that $\phi \in L(m)$, i.e., with

$$
D=\left\{\psi \in M(\mathscr{P} ; \mathbf{R}):|\psi| \leqslant 1, \psi \phi \in L^{1}(m)\right\},
$$

we need by (5.2) and (5.3) to show that $\left\{\int(\psi \phi) \cdot \mathrm{d} m: \psi \in D\right\}$ is bounded in $L^{0}$. Let $\psi \in D$. By (a), (c) and Lemma 3.2 (with $C_{1}>0$ given there), we have

$$
\begin{aligned}
\int_{\mathbf{R}} U\left(s, \psi_{s} \phi_{s}\right) \lambda(\mathrm{d} s) \leqslant \int_{\mathbf{R}} \widetilde{U}\left(s, \psi_{s} \phi_{s}\right) \lambda(\mathrm{d} s) \leqslant \int_{\mathbf{R}} \widetilde{U}\left(s, \phi_{s}\right) \lambda(\mathrm{d} s) \\
\leqslant C_{1}\left[\int_{\mathbf{R}}\left|\left\langle\phi_{s}, b_{s}\right\rangle+\int_{\mathbf{R}^{n}}\left[\tau_{1}\left(\left\langle\phi_{s}, x\right\rangle\right)-\left\langle\phi_{s}, \tau_{n}(x)\right\rangle\right] K(s ; \mathrm{d} x)\right| \lambda(\mathrm{d} s)\right. \\
\left.+\int_{\mathbf{R}} \int_{\mathbf{R}^{n}}\left(1 \wedge\left|\left\langle\phi_{s}, x\right\rangle\right|^{2}\right) K(s ; \mathrm{d} x) \lambda(\mathrm{d} s)\right]<\infty,
\end{aligned}
$$

which shows that

$B(\psi):=\int_{\mathbf{R}}\left(\left\langle\psi_{s} \phi_{s}, b_{s}\right\rangle+\int_{\mathbf{R}^{n}}\left[\tau_{1}\left(\left\langle\psi_{s} \phi_{s}, x\right\rangle\right)-\left\langle\psi_{s} \phi_{s}, \tau_{n}(x)\right\rangle\right] K(s ; \mathrm{d} x)\right) \lambda(\mathrm{d} s)$, is well defined and $\sup _{\psi \in D}|B(\psi)|<\infty$ a.s. In particular, $\{B(\psi): \psi \in D\}$ is bounded in $L^{0}$.

For $\psi \in D$ we have by Lenglart's inequality (see [20, Theorem I]) that for all $\delta, \theta>0$

$$
\begin{aligned}
\mathbf{P}\left(\left|\int(\psi \phi) \cdot \mathrm{d} m^{c}\right| \geqslant \theta\right) & \leqslant \frac{\delta}{\theta^{2}}+\mathbf{P}\left(\int_{\mathbf{R}} \psi_{s}^{2}\left\langle\phi_{s}, c_{s} \phi_{s}\right\rangle \lambda(\mathrm{d} s) \geqslant \delta\right) \\
& \leqslant \frac{\delta}{\theta^{2}}+\mathbf{P}\left(\int_{\mathbf{R}}\left\langle\phi_{s}, c_{s} \phi_{s}\right\rangle \lambda(\mathrm{d} s) \geqslant \delta\right),
\end{aligned}
$$

which by (b) shows that $\left\{\int(\phi \psi) \cdot \mathrm{d} m^{c}: \psi \in D\right\}$ is bounded in $L^{0}$.

Using (c) and the fact that $\left|\tau_{1}(x)\right| \leqslant r(1 \wedge|x|)$ for some $r>0$, Lenglart's inequality shows

$$
\begin{aligned}
& \mathbf{P}\left(\left|\tau_{1}\left(\left\langle x, \psi_{s} \phi_{s}\right\rangle\right) *(\mu-\nu)\right|>\theta\right) \leqslant \frac{\delta}{\theta^{2}}+\mathbf{P}\left(\tau_{1}\left(\left\langle x, \psi_{s} \phi_{s}\right\rangle\right)^{2} * \nu>\delta\right) \\
& \quad \leqslant \frac{\delta}{\theta^{2}}+\mathbf{P}\left(\left(1 \wedge\left\langle x, \psi_{s} \phi_{s}\right\rangle^{2}\right) * \nu>\frac{\delta}{r}\right) \leqslant \frac{\delta}{\theta^{2}}+\mathbf{P}\left(\left(1 \wedge\left\langle x, \phi_{s}\right\rangle^{2}\right) * \nu>\frac{\delta}{r}\right)
\end{aligned}
$$


implying that $\left\{\tau_{1}\left(\left\langle x, \psi_{s} \phi_{s}\right\rangle\right) *(\mu-\nu): \psi \in D\right\}$ is bounded in $L^{0}$.

Let $f(x)=r|x| 1_{\{|x|>\varepsilon\}}$, where $r, \varepsilon>0$ are chosen such that $\left|x-\tau_{1}(x)\right| \leqslant$ $f(x)$. Using that $f$ is symmetric and increasing on $\mathbf{R}_{+}$it follows that

$$
\left|\left(\left\langle x, \psi_{s} \phi_{s}\right\rangle-\tau_{1}\left(\left\langle x, \psi_{s} \phi_{s}\right\rangle\right)\right) * \mu\right| \leqslant f\left(\left\langle x, \psi_{s} \phi_{s}\right\rangle\right) * \mu \leqslant f\left(\left\langle x, \phi_{s}\right\rangle\right) * \mu .
$$

By (c) and Lemma 3.1, $\left(\left|\left\langle x, \phi_{s}\right\rangle\right|^{2} \wedge 1\right) * \mu<\infty$ a.s. In particular, $\sum_{s \in \mathbf{R}} 1_{\left\{\left|\left\langle\Delta m_{s}, \phi_{s}\right\rangle\right|>\varepsilon\right\}}<\infty$ a.s. and hence

$$
f\left(\left\langle x, \phi_{s}\right\rangle\right) * \mu=r \sum_{s \in \mathbf{R}}\left|\left\langle\Delta m_{s}, \phi_{s}\right\rangle\right| 1_{\left\{\left|\left\langle\Delta m_{s}, \phi_{s}\right\rangle\right|>\varepsilon\right\}}<\infty \quad \text { a.s. }
$$

implying that $\left\{\left(\langle x, \psi \phi\rangle-\tau_{1}(\langle x, \psi \phi\rangle)\right) * \mu: \psi \in D\right\}$ is bounded in $L^{0}$.

By (3.4) with $\psi \phi$ playing the role of $\phi$ we have

$$
\begin{aligned}
\int(\psi \phi) \cdot \mathrm{d} m= & \int(\psi \phi) \cdot \mathrm{d} m^{c}+\tau_{1}\left(\left\langle x, \psi_{s} \phi_{s}\right\rangle\right) *(\mu-\nu) \\
& +\left(\left\langle x, \psi_{s} \phi_{s}\right\rangle-\tau_{1}\left(\left\langle x, \psi_{s} \phi_{s}\right\rangle\right)\right) * \mu+B(\psi),
\end{aligned}
$$

and the above shows that the right-hand side is bounded in $L^{0}$ as $\psi$ runs through $D$. Therefore, $\left\{\int(\psi \phi) \cdot \mathrm{d} m: \psi \in D\right\}$ is bounded in $L^{0}$ and the proof is complete.

Before proving Theorem 3.3 we study the relations between convergence in $\mathcal{S M}$ and convergence of triplets. Let $X^{k}=\left(X_{t}^{k}\right)_{t \in \mathbf{R}}$ be real-valued semimartingales up to infinity with $X_{-\infty}^{k}=0$ and let $\left(B^{X^{k}}, C^{X^{k}}, \nu^{X^{k}}\right)$ denote the triplet of $X^{k}$. Write, e.g., $B_{t}^{k}$ instead of $B^{k}((-\infty, t])$ and $B_{\infty}^{k}$ for $B^{k}(\mathbf{R})$.

Theorem 3.5. $X^{k} \rightarrow 0$ in $\mathcal{S M}$ if and only if $\operatorname{Var}\left(B^{X^{k}}\right)_{\infty} \rightarrow 0$, $C_{\infty}^{X^{k}} \rightarrow 0$ and $\left(1 \wedge x^{2}\right) * \nu^{X^{k}} \rightarrow 0$ in $L^{0}$.

For the proof we need the following lemma.

Lemma 3.3. Let $W=W(s, \omega, x)$ be a positive, predictable and bounded function. Then, $W * \mu^{X^{k}} \rightarrow 0$ in $L^{0}$ if and only if $W * \nu^{X^{k}} \rightarrow 0$ in $L^{0}$.

Pro of of $\mathrm{L}$ e m m a 3.3. Assume that $W * \mu^{X^{k}} \rightarrow 0$ in $L^{0}$. For all $k \geqslant 1$ let

$$
\sigma_{k}=\inf \left\{t \in \mathbf{R}: W * \mu_{t}^{X^{k}}>1\right\} .
$$

Assume $W \leqslant c$, then for all $k \geqslant 1, W * \mu_{\sigma_{k}}^{X^{k}} \leqslant 1+c$ and, therefore,

$$
0=\lim _{k \rightarrow \infty} \mathbf{E}\left[W * \mu_{\sigma_{k}}^{X^{k}}\right]=\lim _{k \rightarrow \infty} \mathbf{E}\left[W * \nu_{\sigma_{k}}^{X^{k}}\right] .
$$

For all $\delta>0$ we have by (3.7),

$$
\begin{gathered}
\limsup _{k \rightarrow \infty} \mathbf{P}\left(W * \nu^{X^{k}}>\delta\right) \leqslant \limsup _{k \rightarrow \infty} \mathbf{P}\left(W * \nu_{\sigma_{k}}^{X^{k}}>\delta\right)+\limsup _{k \rightarrow \infty} \mathbf{P}\left(\sigma_{k}<\infty\right) \\
=\limsup _{k \rightarrow \infty} \mathbf{P}\left(\sigma_{k}<\infty\right) \leqslant \limsup _{k \rightarrow \infty} \mathbf{P}\left(W * \mu^{X^{k}}>1\right)=0,
\end{gathered}
$$


which shows that $W * \nu^{X^{k}} \rightarrow 0$ in $L^{0}$. The reverse implication follows similarly. Lemma 3.3 is proved.

$\mathrm{Pr}$ o of of $\mathrm{Th}$ e or e $\mathrm{m}$ 3.5. The if-implication. Assume that the three terms converge in $L^{0}$. For all $t \in \mathbf{R}$,

$$
X_{t}^{k}=X_{t}^{k, c}+\tau_{1}(x) *\left(\mu^{X^{k}}-\nu^{X^{k}}\right)_{t}+\left(x-\tau_{1}(x)\right) * \mu_{t}^{X^{k}}+B_{t}^{X^{k}},
$$

where $X^{k, c}$ denotes the continuous martingale part of $X^{k}$. We shall show that each term on the right-hand side converges to 0 in $\mathcal{S M}$. The total variation of the last term converges to 0 in $L^{0}$, which, since it is predictable, by [21, Theorem IV.7], is equivalent to convergence in $\mathcal{S M}$. By Lenglart's inequality, for all predictable processes $\phi$ with $|\phi| \leqslant 1$ and $\theta, \delta>0$,

$$
\sup _{|\phi| \leqslant 1} \mathbf{P}\left(\left|\int_{-\infty}^{\infty} \phi_{s} \mathrm{~d} X_{s}^{k, c}\right| \geqslant \theta\right) \leqslant \frac{\delta}{\theta^{2}}+\mathbf{P}\left(C_{\infty}^{X^{k}} \geqslant \delta\right)
$$

and with $M_{t}^{k}:=\tau_{1}(x) *\left(\mu^{X^{k}}-\nu^{X^{k}}\right)_{t}$ and using that $\left(\tau_{1}(x)\right)^{2} \leqslant a\left(1 \wedge x^{2}\right)$ for some $a>0$,

$$
\begin{aligned}
\sup _{|\phi| \leqslant 1} \mathbf{P}\left(\left|\int_{-\infty}^{\infty} \phi_{s} \mathrm{~d} M_{s}^{k}\right| \geqslant \theta\right) & \leqslant \frac{\delta}{\theta^{2}}+\mathbf{P}\left(\tau_{1}(x)^{2} * \nu^{X^{k}} \geqslant \delta\right) \\
& \leqslant \frac{\delta}{\theta^{2}}+\mathbf{P}\left(\left(1 \wedge x^{2}\right) * \nu^{X^{k}} \geqslant \frac{\delta}{a}\right) .
\end{aligned}
$$

With $A_{t}^{k}:=\left(x-\tau_{1}(x)\right) * \mu_{t}^{X^{k}}$ and if $\varepsilon, v>0$ are chosen such that $\left|x-\tau_{1}(x)\right| \leqslant$ $v|x| 1_{\{|x|>\varepsilon\}}$, we have

$$
\sup _{|\phi| \leqslant 1}\left|\int_{-\infty}^{\infty} \phi_{s} \mathrm{~d} A_{s}^{k}\right| \leqslant \sup _{|\phi| \leqslant 1} \sum_{s \in \mathbf{R}} v\left|\phi_{s} \Delta X_{s}^{k}\right| 1_{\left\{\left|\Delta X_{s}^{k}\right|>\varepsilon\right\}} \leqslant v \sum_{s \in \mathbf{R}}\left|\Delta X_{s}^{k}\right| 1_{\left\{\left|\Delta X_{s}^{k}\right|>\varepsilon\right\}} .
$$

By Lemma 3.3 and convergence of $\left(1 \wedge x^{2}\right) * \nu^{X^{k}},\left(1 \wedge x^{2}\right) * \mu^{X^{k}} \rightarrow 0$ in $L^{0}$, and therefore for all $\theta \in(0, \varepsilon \wedge 1)$,

$$
\mathbf{P}\left(\sum_{s \in \mathbf{R}}\left|\Delta X_{s}^{k}\right| 1_{\left\{\left|\Delta X_{s}^{k}\right|>\varepsilon\right\}}>\theta\right)=\mathbf{P}\left(\sum_{s \in \mathbf{R}} 1_{\left\{\left|\Delta X_{s}^{k}\right|>\varepsilon\right\}}>\theta\right) \rightarrow 0 .
$$

Thus, all terms on the right-hand side of (3.8) converge to 0 in $\mathcal{S M}$.

To show the only if-part assume that $X^{k} \rightarrow 0$ in $\mathcal{S M}$. By [14], cf. also $\left[12\right.$, p. 307] this implies that the quadratic variation $\left[X^{k}\right]_{\infty}=C_{\infty}^{X^{k}}+x^{2} * \mu^{X^{k}}$ converges to 0 in $L^{0}$. Thus $C_{\infty}^{X^{k}} \rightarrow 0$ and $\left(1 \wedge x^{2}\right) * \mu^{X^{k}} \rightarrow 0$ and therefore by Lemma $3.3\left(1 \wedge x^{2}\right) * \nu^{X^{K}} \rightarrow 0$ in $L^{0}$. It remains to be shown that $\operatorname{Var}\left(B^{X^{k}}\right)_{\infty} \rightarrow 0$ in $L^{0}$. As in (3.9) there exists a constant $l>0$ depending only on the truncation function $\tau_{1}$ such that for $A^{k}$, the third term on the right-hand side of (3.8), $\left\|A^{k}\right\|_{\mathcal{S M}} \leqslant l \mathbf{E}\left[\left[X^{k}\right]_{\infty} \wedge 1\right]$. Thus $A^{k}$, and therefore by linearity the special semimartingales $X^{k}-A^{k}$, converges to 0 in $\mathcal{S M}$. 
Since, cf. [14, p. 273], the map $X \mapsto A(X)$ is continuous in the space of special semimartingales $(A(X)$ denotes the predictable bounded variation component of $X)$, this implies that $B^{X^{k}}=A\left(X^{k}-A^{k}\right) \rightarrow 0$ in $\mathcal{S M}$. But as noted above this means that $\operatorname{Var}\left(B^{X^{k}}\right)_{\infty} \rightarrow 0$ in $L^{0}$. Theorem 3.5 is proved.

As an application of Theorem 3.5 we get the following simple condition for $\mathcal{S} \mathcal{M}$-convergence for Lévy processes.

Corollary 3.1. For all $k \geqslant 1$ let $\left(X_{t}^{k}\right)_{t \in[0,1]}$ be a Lévy process with respect to $\left(\mathcal{F}_{t}\right)_{t \in[0,1]}$ with Lévy-Khintchine triplet $\left(\gamma_{k}, \sigma_{k}^{2}, \kappa_{k}\right)$. Set $X_{t}^{k}=$ $X_{0}^{k}=0$ for $t \leqslant 0$ and $X_{t}^{k}=X_{1}^{k}$ for $t \geqslant 1$. Then, $X^{k} \rightarrow 0$ in $\mathcal{S M}$ if and only if $X_{1}^{k} \rightarrow 0$ in $L^{0}$ (or, equivalently, $\int_{\mathbf{R}}\left(x^{2} \wedge 1\right) \kappa_{k}(\mathrm{~d} x) \rightarrow 0, \sigma_{k}^{2} \rightarrow 0$ and $\left.\gamma_{k} \rightarrow 0\right)$.

P r o o f. By [17, Theorem II.5.15], we have $B_{t}^{k}=\gamma_{k} t, C_{t}^{k}=t \sigma_{k}^{2}$, and $\nu^{k}(\mathrm{~d} s \times \mathrm{d} x)=\kappa_{k}(\mathrm{~d} x) \mathrm{d} s$ for $s, t \in[0,1]$. Hence by Theorem $3.5, X^{k} \rightarrow 0$ in $\mathcal{S M}$ if and only if $\int_{\mathbf{R}}\left(x^{2} \wedge 1\right) \kappa_{k}(\mathrm{~d} x) \rightarrow 0, \sigma_{k}^{2} \rightarrow 0$ and $\left|\gamma_{k}\right| \rightarrow 0$. Moreover, by [27, Theorem 8.7], the latter conditions are equivalent to $X_{1}^{k} \rightarrow 0$ in $L^{0}$. Corollary 3.1 is proved.

Proof of $\mathrm{T}$ h e or e $\mathrm{m}$ 3.3. Assume $\phi^{k} \rightarrow 0$ in $L(m)$, which, as previously noted, is equivalent to $\phi^{k} \bullet m \rightarrow 0$ in $\mathcal{S M}$. If $\left(B^{k}, C^{k}, \nu^{k}\right)$ denotes the triplets of $\phi^{k} \bullet m$, we get by Proposition 3.1 and Theorem 3.5 that for $k \rightarrow \infty$,

$$
\begin{gathered}
\int_{\mathbf{R}} \mid\left\langle\phi_{s}^{k}, b_{s}\right\rangle+\int_{\mathbf{R}^{n}}\left[\tau_{1}\left(\left\langle x, \phi_{s}^{k}\right\rangle\right)\right. \\
\left.-\left\langle\phi_{s}^{k}, \tau_{n}(x)\right\rangle\right] K(s ; \mathrm{d} x) \mid \lambda(\mathrm{d} s) \rightarrow 0 \text { in } L^{0}, \\
\int_{\mathbf{R}}\left\langle\phi_{s}^{k}, c_{s} \phi_{s}^{k}\right\rangle \lambda(\mathrm{d} s) \rightarrow 0 \text { in } L^{0} \\
\int_{\mathbf{R}} \int_{\mathbf{R}^{n}}\left(1 \wedge\left|\left\langle x, \phi_{s}^{k}\right\rangle\right|^{2}\right) K(s ; \mathrm{d} x) \lambda(\mathrm{d} s) \rightarrow 0 \text { in } L^{0}
\end{gathered}
$$

implying $\Psi_{\Phi, 0}\left(\phi^{k}\right) \rightarrow 0$. On the other hand, if $\left\|\phi^{k}\right\|_{\Phi, 0} \rightarrow 0$, then (3.10)(3.12) are satisfied. By Corollary 3.5, $\phi^{k} \bullet m \rightarrow 0$ in $\mathcal{S M}$, showing that $\left\|\phi^{k}\right\|_{m} \rightarrow 0$.

For $x \in \mathbf{R}^{n}$ and $A \in \mathscr{P}$ the above shows in particular that $\left\|x 1_{A}\right\|_{\Phi, 0}=0$ if and only if $\left\|x 1_{A}\right\|_{m}=0$. Thus if $(\mathbf{P} \otimes \lambda)(A)=0$, then for all $x \in \mathbf{R}^{n}$, $\left\|x 1_{A}\right\|_{\Phi, 0}=0$ and hence $\left\|x 1_{A}\right\|_{m}=0$, showing that $A$ is a null-set for $m$. On the other hand, if $A$ is a null-set for $m$, then $\left\|e_{i} 1_{A}\right\|_{m}=0$ for all $i=1, \ldots, n$ ( $e_{i}$ denotes the $i$ th Euclidean standard basic vector) and hence $\left\|e_{i} 1_{A}\right\|_{\Phi, 0}=0$. Therefore, for all $i=1, \ldots, n$, a.s.

$$
0=\int 1_{A}(s) c_{s}^{i, i} \lambda(\mathrm{d} s), \quad 0=\int_{\mathbf{R}} \int_{\mathbf{R}^{n}}\left(1 \wedge\left|1_{A}(s) x^{i}\right|^{2}\right) K(s ; \mathrm{d} x) \lambda(\mathrm{d} s) .
$$

Since $c$ is symmetric and positive semidefinite and $K(s ;\{0\})=0$, equalitites (3.13) show that $\lambda\left(s \in \mathbf{R}: s \in A, c_{s} \neq 0\right)=0$ and $\lambda(s \in \mathbf{R}$ : 
$\left.s \in A, K\left(s ; \mathbf{R}^{n}\right) \neq 0\right)=0$ a.s., and hence, for all $i=1, \ldots, n$,

$$
\begin{aligned}
0= & \int_{\mathbf{R}} \mid\left\langle e_{i} 1_{A}(s), b_{s}\right\rangle \\
& \quad+\int_{\mathbf{R}^{n}}\left[\tau_{1}\left(\left\langle e_{i} 1_{A}(s), x\right\rangle\right)-\left\langle e_{i} 1_{A}(s), \tau_{n}(x)\right\rangle\right] K(s ; \mathrm{d} x) \mid \lambda(\mathrm{d} s) \\
= & \int_{\mathbf{R}}\left|b_{s}^{i} 1_{A}(s)\right| \lambda(\mathrm{d} s) \quad \text { a.s. }
\end{aligned}
$$

Thus if $\lambda\left(s: b_{s}=0, c_{s}=0, K\left(s ; \mathbf{R}^{n}\right)=0\right)=0$ a.s., it follows that $(\lambda \otimes \mathbf{P})(A)=0$. Theorem 3.3 is proved.

4. Lévy processes. In this section $Z=\left\{\left(Z_{t}^{i}\right)_{i \leqslant n}: t \in \mathbf{R}\right\}$ is an $n$ dimensional Lévy process indexed by $\mathbf{R}$ satisfying $Z_{0}=0$ a.s. Let $(\gamma, \Sigma, \kappa)$ denote the Lévy-Khintchine triplet of $Z_{1}$. Our concern is integration with respect to $Z$ in a filtration $\mathscr{F}=\left(\mathcal{F}_{t}\right)_{t \in \mathbf{R}}$ in which $Z$ is an increment semimartingale, that is, induces a Radon measure. Two such filtrations are $\mathscr{F}^{Z \text {,inc }}$ and $\mathscr{F}^{Z}$, given up to completion as

$$
\begin{gathered}
\mathcal{F}_{t}^{Z \text {,inc }}=\sigma\left(Z_{u}-Z_{s}:-\infty<s<u \leqslant t\right), \quad t \in \mathbf{R} . \\
\mathcal{F}_{t}^{Z}=\sigma\left(Z_{u}:-\infty<u \leqslant t\right),
\end{gathered}
$$

(The superscript inc is short for increment). However, a major difference between the two is that $Z$ is a Lévy process in $\mathscr{F}^{Z, \text { inc }}$ but (except in trivial cases) not in $\mathscr{F}^{Z}$. Here we recall that by definition $Z$ is a Lévy process in a filtration $\mathscr{F}$ if, for all $s<t, Z_{t}-Z_{s}$ is independent of $\mathcal{F}_{s}$, measurable with respect to $\mathcal{F}_{t}$, the distribution of $Z_{t}-Z_{s}$ depends only on $t-s$, and $Z$ has càdlàg paths with $Z_{0}=0$. Thus, it is obvious that $Z$ is a Lévy process in $\mathscr{F}^{Z \text {,inc }}$. To see that it is not Lévy in $\mathscr{F}^{Z}$ note that for all $s<0$, $Z_{0}-Z_{s}=-Z_{s}$ is $\mathcal{F}_{s}^{Z}$-measurable and hence not independent of $\mathcal{F}_{s}^{Z}$ except when it is deterministic. Therefore we first consider the case when $Z$ is a Lévy process in $\mathscr{F}$ and then turn to $\mathscr{F}^{Z}$.

4.1. The case when $Z$ is a Lévy process in $\mathscr{F}$. Let $\mathscr{F}$ be a filtration and assume $Z$ is a Lévy process in $\mathscr{F}$. Note that in this case $Z$ is an increment semimartingale in $\mathscr{F}$ and hence induces an $L^{0}$-valued Radon measure as described in Example 3.1. Furthermore, it is easily seen that the triplet of $Z$ is given by

$$
b_{s}=\gamma, \quad c_{s}=\Sigma, \quad K(s ; \mathrm{d} x)=\kappa(\mathrm{d} x), \quad \lambda(\mathrm{d} s)=\mathrm{d} s,
$$

and, therefore, by Theorem 3.2, we have the following corollary.

Corollary 4.1. For all $\mathbf{R}^{n}$-valued $\mathscr{F}$-predictable processes $\phi=\left(\phi_{t}\right)_{t \in \mathbf{R}}$, $\phi \in L(Z)$ if and only if the following (a)-(c) hold almost surely:

(a) $\int_{\mathbf{R}}\left\langle\phi_{s}, \Sigma \phi_{s}\right\rangle \mathrm{d} s<\infty$ 


$$
\begin{aligned}
& \text { (b) } \int_{\mathbf{R}} \int_{\mathbf{R}^{n}}\left(1 \wedge\left|\left\langle\phi_{s}, x\right\rangle\right|^{2}\right) \kappa(\mathrm{d} x) \mathrm{d} s<\infty, \\
& \text { (c) } \int_{\mathbf{R}}\left|\left\langle\gamma, \phi_{s}\right\rangle+\int_{\mathbf{R}^{n}}\left[\tau_{1}\left(\left\langle x, \phi_{s}\right\rangle\right)-\left\langle\phi_{s}, \tau_{n}(x)\right\rangle\right] \kappa(\mathrm{d} x)\right| \mathrm{d} s<\infty .
\end{aligned}
$$

Consider the special case, where $Z$ is an $\mathbf{R}^{n}$-valued strictly $\alpha$-stable Lévy process with $\alpha \in(0,2)$. In this case the conditions in Corollary 4.1 become particularly simple. Since $\Sigma=0$, only (b) and (c) have to be verified. According to [27, Theorem 14.3], there exists a finite measure $\Theta$, often referred to as the spherical part of $\kappa$, on $S^{n-1}=\left\{x \in \mathbf{R}^{n}:\|x\|=1\right\}$, such that the Lévy measure $\kappa$ of $Z$ is of the form

$$
\kappa(A)=\int_{S^{n-1}}\left(\int_{0}^{\infty} \frac{1_{A}(r x)}{r^{1+\alpha}} \mathrm{d} r\right) \Theta(\mathrm{d} x), \quad A \in \mathscr{B}\left(\mathbf{R}^{n}\right) .
$$

The following corollary extends results of [25] and [11] to processes indexed by $\mathbf{R}$ and with values in $\mathbf{R}^{n}$.

Corollary 4.2. Let $Z=\left(Z_{t}\right)_{t \in \mathbf{R}}$ be an $\mathbf{R}^{n}$-valued strictly $\alpha$-stable Lévy process with Lévy measure given by (4.1) (for $\alpha=1$, assume $Z$ is symmetric, which in particular implies $\gamma=0)$. Then for all $\mathscr{F}$-predictable $\mathbf{R}^{n}$-valued processes $\phi$, the integral $\int_{\mathbf{R}} \phi_{s} \cdot \mathrm{d} Z_{s}$ exists if and only if

$$
\int_{\mathbf{R}}\left(\int_{S^{n-1}}\left|\left\langle\phi_{s}, x\right\rangle\right|^{\alpha} \Theta(\mathrm{d} x)\right) \mathrm{d} s<\infty \quad \text { a.s. }
$$

$\mathrm{P} \mathrm{r}$ o o f. For simplicity let $\tau_{n}$ and $\tau_{1}$ be the truncation functions on $\mathbf{R}^{n}$ and $\mathbf{R}$ given by $\tau_{n}(x)=x 1_{\{\|x\| \leqslant 1\}}$ and $\tau_{1}(x)=x 1_{\{|x| \leqslant 1\}}$. Due to the fact that $Z$ is strictly stable we have $\gamma=\int_{\mathbf{R}^{n}}\left(\tau_{n}(x)-x\right) \kappa(\mathrm{d} x)$ for $\alpha \in(1,2)$ and $\gamma=\int_{\mathbf{R}^{n}} \tau_{n}(x) \kappa(\mathrm{d} x)$ for $\alpha \in(0,1)$, cf. [27, Theorem 14.7]. Using (4.1), a simple calculation shows

$$
\begin{aligned}
C_{1, \alpha} & \int_{\mathbf{R}^{n}}\left(1 \wedge\left|\left\langle\phi_{s}, x\right\rangle\right|^{2}\right) \kappa(\mathrm{d} x)=\int_{S^{n-1}}\left|\left\langle\phi_{s}, x\right\rangle\right|^{\alpha} \Theta(\mathrm{d} x) \\
& \geqslant C_{2, \alpha}\left|\left\langle\phi_{s}, \gamma\right\rangle+\int_{\mathbf{R}^{n}}\left[\tau_{1}\left(\left\langle\phi_{s}, x\right\rangle\right)-\left\langle\phi_{s}, \tau_{n}(x)\right\rangle\right] \kappa(\mathrm{d} x)\right|,
\end{aligned}
$$

where equality (4.3) holds for all $\alpha \in(0,2)$ and $C_{1, \alpha}=(2-\alpha) \alpha / 2$, and inequality (4.4) holds for $\alpha \neq 1$ and $C_{2, \alpha}=|\alpha-1|$. In the case $\alpha=1$ inequality (4.4) remains true for some constant $C_{2,1}$. This shows that (a)(c) of Corollary 4.1 are equivalent to (4.2). Corollary 4.2 is proved.

4.2. Integrability in $\mathscr{F}^{Z}$. When considering integrals as $(1.2)(\beta)$ the process $\left\{f\left(s, Z_{s-}\right): s \in \mathbf{R}\right\}$ is usually not predictable (nor adapted) in $\mathscr{F}^{Z}$,inc (or in any other filtration in which $Z$ is a Lévy process). Thus, $(1.2)(\beta)$ cannot be studied in $\mathscr{F}^{Z \text {,inc }}$, forcing us to consider instead the filtration $\mathscr{F}^{Z}$. Although $Z$ is an increment semimartingale in $\mathscr{F}^{Z}$ (by Theorem 4.1 below and Stricker's theorem), it is in general difficult to calculate its triplet in 
this filtration. Therefore we consider the larger filtration $\mathscr{F}^{Z \text {,ex }}$ given by $\mathcal{F}_{t}^{Z \text {,ex }}=\mathcal{F}_{t}^{Z}$ for $t \geqslant 0$ and

$$
\mathcal{F}_{t}^{Z \text {,ex }}=\mathcal{F}_{t}^{Z} \vee \sigma\left(\mu^{Z}((t, 0] \times A): A \in \mathscr{B}\left(\mathbf{R}^{n}\right)\right) \quad \text { for } t<0,
$$

in which we are able to calculate the triplet explicitly. (The superscript ex is short for extended.) Note that $\mathcal{F}_{t}^{Z \text {,inc }} \subseteq \mathcal{F}_{t}^{Z} \subseteq \mathcal{F}_{t}^{Z \text {,ex }}$ for all $t \in \mathbf{R}$. Let $Z^{c}=\left(Z_{t}^{c}\right)_{t \in \mathbf{R}}$ be the Gaussian component of $Z$ chosen such that $Z_{0}=0$. Recall that $\mu^{Z}$ is the jump measure of $Z$, and set $\nu(\mathrm{d} s \times \mathrm{d} x)=\kappa(\mathrm{d} x) \mathrm{d} s$ and

$$
M_{t}= \begin{cases}Z_{t}^{c}+\int_{0}^{t} \int_{\mathbf{R}^{n}} \tau_{n}(x)\left(\mu^{Z}-\nu\right)(\mathrm{d} s \times \mathrm{d} x), & t \geqslant 0, \\ Z_{t}^{c}-\int_{t}^{0} \int_{\mathbf{R}^{n}} \tau_{n}(x)\left(\mu^{Z}-\nu\right)(\mathrm{d} s \times \mathrm{d} x), & t<0 .\end{cases}
$$

Due to the fact that $\mathcal{F}_{t}^{Z} \subseteq \mathcal{F}_{t}^{Z \text {,ex }}$ for all $t \in \mathbf{R}$, the following result gives in particular sufficient conditions for the integral $\int_{\mathbf{R}} \phi_{s} \cdot \mathrm{d} Z_{s}$ to exist for any $\mathscr{F}^{Z}$-predictable process $\phi$.

Theorem 4.1. In the filtration $\mathscr{F}^{Z, \mathrm{ex}}, Z$ is an increment semimartingale with triplet

$$
\begin{gathered}
b_{s}=\gamma+\frac{M_{s}}{s} 1_{\{s<0\}}, \quad c_{s}=\Sigma, \quad \lambda(\mathrm{d} s)=\mathrm{d} s, \\
K(s ; \mathrm{d} x)=\frac{\mu^{Z}((s, 0] \times \mathrm{d} x)}{|s|} 1_{\{s<0\}}+\kappa(\mathrm{d} x) 1_{\{s \geqslant 0\}} .
\end{gathered}
$$

Hence for all $\mathscr{F}^{Z, \mathrm{ex}}$-predictable processes $\phi$, the integral $\int_{\mathbf{R}} \phi_{s} \cdot \mathrm{d} Z_{s}$ exists if and only if (3.5) and (3.6) are satisfied with $b, c, \lambda$, and $K$ given by (4.5) and (4.6).

Note that the triplet of $Z$ with respect to $\mathscr{F}^{Z \text {,ex }}$ is random, reflecting that $Z$ does not have independent increments with respect to $\mathscr{F}^{Z \text {,ex }}$. Note also that for all $s<0, \mu^{Z}((s, 0] \times \mathrm{d} x)$ is a Poisson random measure with intensity measure $|s| \kappa(\mathrm{d} x)$. Finally, when $Z$ is Gaussian we have $\mathscr{F}^{Z \text {,ex }}=$ $\mathscr{F}^{Z}$ so in this case the above result gives necessary and sufficient conditions for existence of the integral in $\mathscr{F}^{Z}$.

Theorem 4.1 relies on the following expansion of filtration result which extends Theorems 2.6 and 2.9 in [16] to the multivariate case.

Theorem 4.2. Let $t_{0}>0$ be fixed and let $\mathcal{H}=\left(\mathcal{H}_{t}\right)_{t \geqslant 0}$ be the least filtration to which $\left(Z_{t}\right)_{t \geqslant 0}$ is adapted and satisfying for all Borel sets $A$ bounded away from zero that $\mu_{Z}\left(\left(0, t_{0}\right] \times A\right)$ and $Z_{t_{0}}$ are $\mathcal{H}_{t}$-measurable for all $t \geqslant 0$. Then $\left(Z_{t}\right)_{t \geqslant 0}$ is a semimartingale with respect to $\mathcal{H}$ with triplet

$$
\begin{gathered}
b_{s}=\gamma+\frac{M_{t_{0}}-M_{s}}{t_{0}-s} 1_{\left\{0 \leqslant s<t_{0}\right\}}, \quad c_{s}=\Sigma, \quad \lambda(\mathrm{d} s)=\mathrm{d} s, \\
K(s ; \mathrm{d} x)=\frac{\mu^{Z}\left(\left(s, t_{0}\right] \times \mathrm{d} x\right)}{t_{0}-s} 1_{\left\{0 \leqslant s<t_{0}\right\}}+\kappa(\mathrm{d} x) 1_{\left\{s \geqslant t_{0}\right\}} .
\end{gathered}
$$


To prove Theorem 4.2 one cannot apply the theorems in [16] coordinatewise. However, for integrable independent and identically distributed $\mathbf{R}^{n}$-valued random vectors $Y_{i}, i=1, \ldots, k$, we have $\mathbf{E}\left[Y_{1} \mid \sum_{i=1}^{k} Y_{i}\right]=$ $(1 / k) \sum_{i=1}^{k} Y_{i}$. Using this, Theorem 4.2 follows along the lines of the proofs in [16]; see [6, Appendix A] for details.

Proof of $\mathrm{Th}$ e or e m 4.1. Fix an $r<0$ and define $Z^{(r)}=$ $\left(Z_{t}^{(r)}\right)_{t \geqslant r}$ as $Z_{t}^{(r)}=Z_{t}-Z_{r}$. Consider the filtration $\mathscr{F}^{Z^{(r)}, \text { ex }}=\left(\mathcal{F}_{t}^{Z^{(r)}, \text { ex }}\right)_{t \geqslant r}$, where

$$
\mathcal{F}_{t}^{Z^{(r)}, \mathrm{ex}}=\sigma\left(Z_{u}^{(r)}: u \in[r, t]\right) \vee \sigma\left(Z_{0}^{(r)}\right) \vee \sigma\left(\mu^{Z^{(r)}}((r, 0] \times A): A \in \mathscr{B}\left(\mathbf{R}^{n}\right)\right) .
$$

Using that $Z_{r}=-\left(Z_{0}-Z_{r}\right)=-Z_{0}^{(r)}$, we get for $t \geqslant r$,

$$
\mathcal{F}_{t}^{Z \text {,ex }}=\mathscr{F}_{t}^{Z^{(r)}, \text { ex }} \vee \mathcal{G}_{r}, \quad \text { where } \quad \mathcal{G}_{r}=\sigma\left(Z_{r}-Z_{u}: u<r\right) .
$$

By Theorem 4.2 (where $r$ plays the role of 0 , and 0 plays the role of $t_{0}$ ), $Z^{(r)}$ is a semimartingale with respect to $\mathscr{F}^{Z^{(r)}}$,ex . Let $b^{(r)}, c^{(r)}, K^{(r)}$, and $\lambda^{(r)}$ denote the quantities defining the triplet of $Z^{(r)}$ in (4.7) and (4.8). Let $Z^{(r), c}$ denote the Gaussian component of the Lévy process $Z^{(r)}$ and note that $Z_{t}^{(r), c}=Z_{t}^{c}-Z_{r}^{c}$. Thus, with

$$
\begin{aligned}
M_{t}^{(r)}: & =Z_{t}^{(r), c}+\int_{(r, t] \times \mathbf{R}^{n}} \tau_{n}(x)\left(\mu^{Z^{(r)}}-\nu\right)(\mathrm{d} s \times \mathrm{d} x) \\
& =\left(Z_{t}^{c}-Z_{r}^{c}\right)+\int_{(r, t] \times \mathbf{R}^{n}} \tau_{n}(x)\left(\mu^{Z}-\nu\right)(\mathrm{d} s \times \mathrm{d} x), \quad t \geqslant r,
\end{aligned}
$$

we have by Theorem 4.2 that

$$
b_{s}^{(r)}=\gamma+\frac{M_{0}^{(r)}-M_{s}^{(r)}}{0-s} 1_{\{r \leqslant s<0\}}=\gamma+\frac{M_{s}}{s} 1_{\{r \leqslant s<0\}} .
$$

Similarly,

$$
\begin{aligned}
K^{(r)}(s ; \mathrm{d} x) & =\frac{\mu^{Z^{(r)}}((s, 0] \times \mathrm{d} x)}{0-s} 1_{\{r \leqslant s \leqslant 0\}}+\kappa(\mathrm{d} x) 1_{\{s \geqslant 0\}} \\
& =\frac{\mu^{Z}((s, 0] \times \mathrm{d} x)}{|s|} 1_{\{r \leqslant s \leqslant 0\}}+\kappa(\mathrm{d} x) 1_{\{s \geqslant 0\}} .
\end{aligned}
$$

Finally, $\lambda^{(r)}(\mathrm{d} s)=1_{\{s \geqslant r\}} \mathrm{d} s$ and $c_{s}=\Sigma$. Since $\mathcal{G}_{r}$ is independent of $Z^{(r)}=$ $\left(Z_{t}-Z_{r}\right)_{t \geqslant r}$, it follows by (4.9) that $Z^{(r)}$ is an $\left(\mathcal{F}_{t}^{Z, \text { ex }}\right)_{t \geqslant r}$-semimartingale with the above triplet. Furthermore, since this is true for all $r<0$, it follows that the triplet of $Z$ is given by (4.5)-(4.6). Theorem 4.1 is proved.

$\mathrm{R}$ e $\mathrm{m}$ a r k 4.1. Even though $b_{s}$ and $K(s ; \mathrm{d} x)$ are random for $s \in$ $(-\infty, 0)$ it follows by the strong law of large numbers for Lévy processes (see, e.g., [27, Theorem 36.5]) that they are both deterministic in the limit $s \rightarrow$ 
$-\infty$; in fact, for all measurable functions $f: \mathbf{R}^{n} \rightarrow \mathbf{R}_{+}, \int_{\mathbf{R}^{n}} f(x) K(s ; \mathrm{d} x) \rightarrow$ $\int_{\mathbf{R}^{n}} f(x) \kappa(\mathrm{d} x)$ and $b_{s} \rightarrow \gamma$ a.s. as $s \rightarrow-\infty$.

In the following result we use Remark 4.1 to give a simple condition that $\int_{\mathbf{R}} \phi_{s} \cdot \mathrm{d} Z_{s}$ exists.

Theorem 4.3. Assume $\mathbf{E}\left[\left\|Z_{1}\right\|^{2}\right]<\infty$. Then, for all $\mathscr{F}^{Z}$-predictable processes $\phi$ with a.s. locally bounded sample paths the integral $\int_{\mathbf{R}} \phi_{s} \cdot \mathrm{d} Z_{s}$ exists if $\int_{\mathbf{R}}\left(\left\|\phi_{s}\right\|^{2}+\left\|\phi_{s}\right\|\right) \mathrm{d} s<\infty$ a.s.

P r o o f. Let us show that (a)-(c) of Theorem 3.2 are satisfied with $b, c, K$, and $\lambda$ as in Theorem 4.1. Property (b) follows from the fact that $\left\langle\phi_{s}, \Sigma \phi_{s}\right\rangle \leqslant C_{\Sigma}\left\|\phi_{s}\right\|^{2}$ for some constant $C_{\Sigma} \geqslant 0$ depending only on $\Sigma$.

In the proof of (a) and (c) we will use the following consequences of Remark 4.1. As $s \rightarrow-\infty$ we have that

$$
\begin{aligned}
\int_{\mathbf{R}^{n}}\|x\|^{2} K(s ; \mathrm{d} x) & \longrightarrow \int_{\mathbf{R}^{n}}\|x\|^{2} \kappa(\mathrm{d} x) \quad \text { a.s. } \\
\int_{\{\|x\|>1\}}\|x\| K(s ; \mathrm{d} x) & \longrightarrow \int_{\{\|x\|>1\}}\|x\| \kappa(\mathrm{d} x) \quad \text { a.s. } \\
b_{s} & \longrightarrow \gamma \quad \text { a.s. }
\end{aligned}
$$

For simplicity let $\tau_{n}(x)=x 1_{\{\|x\| \leqslant 1\}}$ for $x \in \mathbf{R}^{n}$ and $\tau_{1}(y)=y 1_{\{|y| \leqslant 1\}}$ for $y \in \mathbf{R}$. To verify (c) it suffices, due to the fact that $\phi$ has a.s. locally bounded sample paths, to show that there exists a $k=k(\omega) \in \mathbb{N}$ such that

$$
\int_{[-k, k]^{c}} \int_{\mathbf{R}^{n}}\left(1 \wedge\left\langle\phi_{s}, x\right\rangle^{2}\right) K(s ; \mathrm{d} x) \mathrm{d} s<\infty .
$$

However, since

$$
\int_{\mathbf{R}^{n}}\left(1 \wedge\left|\left\langle\phi_{s}, x\right\rangle\right|^{2}\right) K(s ; \mathrm{d} x) \leqslant\left\|\phi_{s}\right\|^{2} \int_{\mathbf{R}^{n}}\|x\|^{2} K(s ; \mathrm{d} x),
$$

equation (4.10) implies (4.13). To show (a) note that

$$
\begin{aligned}
\left|\tau_{1}\left(\left\langle\phi_{s}, x\right\rangle\right)-\left\langle\phi_{s}, \tau_{n}(x)\right\rangle\right| & =\left|\left\langle\phi_{s}, x\right\rangle\left(1_{\left\{\left|\left\langle\phi_{s}, x\right\rangle\right| \leqslant 1\right\}}-1_{\{\|x\| \leqslant 1\}}\right)\right| \\
& \leqslant\left\|\phi_{s}\right\|^{2}\|x\|^{2}+\left\|\phi_{s}\right\|\|x\| 1_{\{\|x\|>1\}},
\end{aligned}
$$

and therefore (a) follows in the same way as (c) using (4.10)-(4.12) and the estimates

$$
\begin{aligned}
& \left|\left\langle\phi_{s}, b_{s}\right\rangle+\int_{\mathbf{R}^{n}}\left[\tau_{1}\left(\left\langle\phi_{s}, x\right\rangle\right)-\left\langle\phi_{s}, \tau_{n}(x)\right\rangle\right] K(s ; \mathrm{d} x)\right| \\
& \quad \leqslant\left\|\phi_{s}\right\|\left\|b_{s}\right\|+\int_{\mathbf{R}^{n}}\left|\tau_{1}\left(\left\langle\phi_{s}, x\right\rangle\right)-\left\langle\phi_{s}, \tau_{n}(x)\right\rangle\right| K(s ; \mathrm{d} x) \\
& \quad \leqslant\left\|\phi_{s}\right\|\left\|b_{s}\right\|+\left\|\phi_{s}\right\|^{2} \int_{\mathbf{R}^{n}}\|x\|^{2} K(s ; \mathrm{d} x)+\left\|\phi_{s}\right\| \int_{\{\|x\|>1\}}\|x\| K(s ; \mathrm{d} x) .
\end{aligned}
$$

Theorem 4.3 is proved. 
5. Appendix: Vector measures. Let $(E, \mathscr{E})$ denote a measurable space and for all $n \geqslant 1$ let $M\left(\mathscr{E} ; \mathbf{R}^{n}\right)$ be the space of all $\mathbf{R}^{n}$-valued $\mathscr{E}$ measurable functions. Furthermore, $(F,\|\cdot\|)$ denotes an $F$-space in which unconditional convergence implies bounded multiplier convergence (see [24]); i.e., for all $\varepsilon>0$ there exists a $\delta>0$ such that for all $k \geqslant 1$ and $x_{1}, \ldots, x_{k} \in F$

$$
\begin{aligned}
\left\|\sum_{i=1}^{k} \varepsilon_{i} x_{i}\right\|<\delta \text { for all }\left(\varepsilon_{i}\right)_{i=1}^{k} \subseteq\{0,1\} \\
\Rightarrow \quad\left\|\sum_{i=1}^{k} a_{i} x_{i}\right\|<\varepsilon \text { for all }\left(a_{i}\right)_{i=1}^{k} \subseteq[-1,1] .
\end{aligned}
$$

Here we follow Rolewicz [24] and call $(F,\|\cdot\|)$ an $F$-space if and only if $d(x, y):=\|x-y\|$ defines a metric in which $F$ is a linear complete metric space. We may and do always assume that $\|\cdot\|$ is increasing, that is, for all $a \in[-1,1],\|a x\| \leqslant\|x\|$ (cf. [24, Theorem I.2.2]).

This covers in particular $F=L^{0}(\Omega, \mathcal{F}, \mathbf{P})$, the space of real-valued random variables on a probability space $(\Omega, \mathcal{F}, \mathbf{P})$, equipped with the $F$ norm $\|Z\|_{0}:=\mathbf{E}[|Z| \wedge 1]$, cf. [26], together with all locally convex $F$-spaces (e.g., Banach spaces), cf. [24, Corollary III.6.6]. In the following we extend the well-known theory of integration with respect to finite vector measures to $\sigma$-finite measures; this extension is slight but important, e.g., since increment martingales induce $\sigma$-finite measures.

5.1. The one-dimensional case. We call a set function $m$ defined on a subset of $\mathscr{E}$ an $F$-valued $\sigma$-finite measure on $(E, \mathscr{E})$ if there exists a sequence $\left(O_{k}\right)_{k \geqslant 1} \subseteq \mathscr{E}$ with $O_{k} \uparrow E$ such that, with $\mathscr{E}_{m}=\{A \in \mathscr{E}: A \subseteq$ $O_{k}$ for some $\left.k \geqslant 1\right\}$, the mapping $m: \mathscr{E}_{m} \rightarrow F$ satisfies $m(\varnothing)=0$ and if $\left(A_{i}\right)_{i=1}^{\infty} \subseteq \mathscr{E}_{m}$ are disjoint sets with $\bigcup_{i=1}^{\infty} A_{i} \in \mathscr{E}_{m}$, then $m\left(\bigcup_{i=1}^{\infty} A_{i}\right)=$ $\sum_{i=1}^{\infty} m\left(A_{i}\right)$ in $F$. The sequence $\left(O_{k}\right)_{k \geqslant 1}$ is called $m$-feasible. Denote by $\mathscr{S}_{m}$ the vector space of all real-valued $\mathscr{E}_{m}$-simple functions, i.e., $\phi \in \mathscr{S}_{m}$ if and only if

$$
\phi=\sum_{i=1}^{k} a_{i} 1_{A_{i}} \quad \text { for some } a_{1}, \ldots, a_{k} \in \mathbf{R} \text { and } A_{1}, \ldots, A_{k} \in \mathscr{E}_{m}
$$

Clearly the $A_{i}$ 's may be assumed disjoint. Set for $\phi \in \mathscr{S}_{m}$ of the form (5.1) $m(\phi)=\sum_{i=1}^{k} a_{i} m\left(A_{i}\right)$. Then $m: \mathscr{S}_{m} \rightarrow F$ is linear and is called the simple integral with respect to $m$. We are looking for a linear extension $\int \cdot \mathrm{d} m$ of the simple integral defined on a vector space $\mathscr{D} \subseteq E^{\mathbf{R}}$ satisfying the DCT (Dominated Convergence Theorem), i.e., whenever $\left(\phi_{k}\right)_{k \geqslant 1}, \psi \subseteq \mathscr{D}$, with $\left|\phi_{k}\right| \leqslant \psi$ and $\phi_{k} \rightarrow \phi$ pointwise, we have $\phi \in \mathscr{D}$ and $\int \phi_{k} \mathrm{~d} m \rightarrow \int \phi \mathrm{d} m$ in $F$. Note that the DCT ensures that every bounded $\mathscr{E}$-measurable function vanishing outside some $O_{k}$ is in $\mathscr{D}$. 
In order to state the main theorem we introduce the semivariation $\|\cdot\|_{m}$ of $m$, that is,

$$
\|\phi\|_{m}=\sup _{\psi \in \mathscr{S}_{m}:|\psi| \leqslant|\phi|}\|m(\psi)\| \quad \text { for } \phi \in M(\mathscr{E} ; \mathbf{R}) .
$$

It is readily seen that: (i) $\left|\phi_{1}\right| \leqslant\left|\phi_{2}\right|$ implies $\left\|\phi_{1}\right\|_{m} \leqslant\left\|\phi_{2}\right\|_{m}$; (ii) $\|\cdot\|_{m}$ is subadditive, i.e., $\left\|\phi_{1}+\phi_{2}\right\|_{m} \leqslant\left\|\phi_{1}\right\|_{m}+\left\|\phi_{2}\right\|_{m}$; (iii) $\|m(\phi)\| \leqslant\|\phi\|_{m}$ for $\phi \in \mathscr{S}_{m}$.

Set, furthermore,

$$
L^{1}(m)=\left\{\phi \in M(\mathscr{E} ; \mathbf{R}): \lim _{\lambda \rightarrow 0}\|\lambda \phi\|_{m}=0\right\}
$$

or, equivalently,

$$
L^{1}(m)=\left\{\phi \in M(\mathscr{E} ; \mathbf{R}):\left\{m(\psi):|\psi| \leqslant|\phi|, \psi \in \mathscr{S}_{m}\right\} \text { is bounded in } F\right\} .
$$

Note that $\|\cdot\|_{m}$, and hence $L^{1}(m)$, is invariant of the choice of $\left(O_{k}\right)_{k \geqslant 1}$. Thus, it is $L^{1}(m)$ rather than $\left(O_{k}\right)_{k \geqslant 1}$ that is important. Indeed, if $\left(\widetilde{O}_{k}\right)_{k \geqslant 1} \subseteq \mathscr{E}$ with $\widetilde{O}_{k} \uparrow E$ is another $m$-feasible sequence then the two semivariations agree; that is,

$$
\|\phi\|_{m}=\sup _{\psi \in \widetilde{\mathscr{S}}_{m}:|\psi| \leqslant|\phi|}\|m(\psi)\| \quad \text { for } \phi \in M(\mathscr{E} ; \mathbf{R}) .
$$

Here $\widetilde{\mathscr{S}}_{m}$ denotes the simple functions relative to $\left(\widetilde{O}_{k}\right)_{k \geqslant 1}$. To see this, we may and do assume $\widetilde{O}_{k} \subseteq O_{k}$ for all $k \geqslant 1$, implying that the left-hand side dominates the right-hand side. To get the other inequality observe that $A=\bigcup_{k=1}^{\infty} A \cap\left(\widetilde{O}_{k} \backslash \widetilde{O}_{k-1}\right)$ for $A \in \mathscr{E}_{m}$. The $\sigma$-additivity of $m$ on each $O_{k}$ therefore ensures that for all $\psi \in \mathscr{S}_{m}$ there exists a sequence $\left(\psi_{k}\right)_{k \geqslant 1} \subseteq \widetilde{\mathscr{S}}_{m}$ with $\left|\psi_{k}\right| \leqslant|\psi|$ for all $k$ such that $m\left(\psi_{k}\right) \rightarrow m(\psi)$ in $F$. But this means that $\left\|m\left(\psi_{k}\right)\right\| \rightarrow\|m(\psi)\|$, proving the result.

Theorem 5.1. Assume that $m$ is locally bounded, i.e., the set $\left\{m(B): B \in \mathcal{E} \cap O_{k}\right\}$ is bounded in $F$ for all $k \geqslant 1$. Then $L^{1}(m)$ is a linear space and equipped with $\|\cdot\|_{m}$ it is an $F$-space containing $\mathscr{S}_{m}$ as a dense subspace. The simple integral extends to $L^{1}(m)$ by $\|\cdot\|_{m}$-continuity and the extension $\int \cdot \mathrm{d} m: L^{1}(m) \rightarrow F$ satisfies the DCT. More generally, if $\left(\phi_{k}\right)_{k \geqslant 1} \subseteq L^{1}(m), \phi_{k} \rightarrow \phi$ pointwise and there exists $\psi \in L^{1}(m)$ such that $\left|\phi_{k}\right| \leqslant \psi$ for all $k \geqslant 1$, then $\phi_{k} \rightarrow \phi$ in $L^{1}(m)$.

Moreover, $\phi \in L^{1}(m)$ if and only if there exists $\left(\phi_{k}\right)_{k \geqslant 1} \subseteq \mathscr{S}_{m}$ such that $\phi_{k} \rightarrow \phi$ pointwise and for all $A \in \mathscr{E}, \lim _{k} \int_{A} \phi_{k} \mathrm{~d} m$ exists in $F$.

$\mathrm{R}$ e $\mathrm{m}$ a r k 5.1. In the important case $F=L^{0}(\Omega, \mathcal{F}, \mathbf{P})$ all $\sigma$-finite measures are locally bounded, cf. [29].

P r o of of $\mathrm{T}$ h e or e m 5.1. It suffices to verify $\left(E_{1}\right)-\left(E_{3}\right)$ in $[8$, p. 278]. This can, however, be done by following Kwapień and Woyczyński 
[19, Theorem 7.1.2] who consider the case $F=L^{0}$ and a finite rather than a $\sigma$-finite measure; see also [24, Theorem III.6.2]. For details of the proof see [6, Theorem 2.1]. Theorem 5.1 is proved.

When $1_{E} \in L^{1}(m)$ we call $m$ a finite measure, and in this case $m$ extends to a measure defined on the entire $\sigma$-field $\mathscr{E}$.

5.2. The multivariate case. In the following we define and study the integral with respect to $m=\left(m^{i}\right)_{i \leqslant n}$, where $m^{1}, \ldots, m^{n}$ are $F$-valued $\sigma$-finite and locally bounded measures as defined in Subsection 5.1. Let $\left(O_{k}\right)_{k \geqslant 1} \subseteq \mathscr{E}$ with $O_{k} \uparrow E$ be a sequence which is $m^{i}$-feasible for all $i$. Our construction of the integral is motivated by [10, Section 3].

Setting

$$
L^{1}(m)=\left\{\phi=\left(\phi^{i}\right)_{i=1}^{n} \in M\left(\mathscr{E} ; \mathbf{R}^{n}\right): \phi^{i} \in L^{1}\left(m^{i}\right) \text { for all } i=1, \ldots, n\right\}
$$

gives a linear space stable under multiplication with bounded elements in $M(\mathscr{E} ; \mathbf{R})$. Define for $\phi \in L^{1}(m)$ the integral of $\phi$ with respect to $m$ as $\int \phi \cdot \mathrm{d} m=\sum_{i=1}^{n} \int \phi^{i} \mathrm{~d} m^{i}$. Set for $\phi \in M\left(\mathscr{E} ; \mathbf{R}^{n}\right)$,

$$
\|\phi\|_{m}=\sup \left\{\left\|\int(\phi \psi) \cdot \mathrm{d} m\right\|: \psi \in M(\mathscr{E} ; \mathbf{R}),|\psi| \leqslant 1, \psi \phi \in L^{1}(m)\right\},
$$

and let

$$
L(m)=\left\{\phi \in M\left(\mathscr{E} ; \mathbf{R}^{n}\right): \lim _{\lambda \rightarrow 0}\|\lambda \phi\|_{m}=0\right\} .
$$

Fix $\phi \in M\left(\mathscr{E} ; \mathbf{R}^{n}\right)$ and set $\bar{O}_{k}=O_{k} \cap\{\|\phi\| \leqslant k\}$ for $k \geqslant 1$, and $\mathscr{E}_{\phi \bullet m}=\left\{A \in \mathscr{E}: A \subseteq \bar{O}_{k}\right.$ for some $\left.k \geqslant 1\right\}$. Then, $\phi \bullet m: \mathscr{E}_{\phi \bullet m} \rightarrow F$ defined as $\phi \bullet m(A)=\int\left(1_{A} \phi\right) \cdot \mathrm{d} m$ is an $F$-valued $\sigma$-finite and locally bounded measure on $(E, \mathscr{E})$. Note that $\phi \in L^{1}(m)$ implies $1_{E} \in L^{1}(\phi \bullet m)$ and hence, applying the DCT of $\phi \bullet m$ and the $m^{i}$ s, we get in this case

$$
\phi \bullet m(E)=\lim _{k \rightarrow \infty} \phi \bullet m\left(\bar{O}_{k}\right)=\lim _{k \rightarrow \infty} \int\left(1_{\bar{O}_{k}} \phi\right) \cdot \mathrm{d} m=\int \phi \cdot \mathrm{d} m,
$$

which motivates the following definition.

D e f i n it i o n 5.1. $\phi \in M\left(\mathscr{E} ; \mathbf{R}^{n}\right)$ is said to be integrable with respect to $m$ if $1_{E} \in L^{1}(\phi \bullet m)$, and in this case $\int \phi \cdot \mathrm{d} m:=\phi \bullet m(E) \in F$ is the integral of $\phi$ with respect to $m$.

The following gives the basic properties (recall that an $F^{*}$-space is an $F$-space except that it is not necessarily complete).

Theorem 5.2. $\left(L(m),\|\cdot\|_{m}\right)$ is an $F^{*}$-space, $\phi$ is integrable with respect to $m$ if and only if $\phi \in L(m)$, and the mapping $L(m) \ni \phi \mapsto \int \phi \cdot \mathrm{d} m \in F$ is linear. Moreover, for $\phi \in M\left(\mathscr{E} ; \mathbf{R}^{n}\right)$ and $\psi \in M(\mathscr{E} ; \mathbf{R})$ we have $\psi \in$ $L^{1}(\phi \bullet m)$ if and only if $\psi \phi \in L(m)$ and

$$
\int \psi \mathrm{d}(\phi \bullet m)=\int(\psi \phi) \cdot \mathrm{d} m \quad \text { for } \phi \in M\left(\mathscr{E} ; \mathbf{R}^{n}\right) \text { and } \psi \in L^{1}(\phi \bullet m) .
$$


P r o o f. For $\phi_{1}, \phi_{2} \in M\left(\mathscr{E} ; \mathbf{R}^{n}\right)$ we can, using the DCT, show that $\left\|\phi_{1}+\phi_{2}\right\|_{m} \leqslant\left\|\phi_{1}\right\|_{m}+\left\|\phi_{2}\right\|_{m}$ and hence it follows that $L(m)$ is an $F^{*}$-space. The last part of Theorem 5.2 follows once we have shown that $\|\psi\|_{\phi \bullet m}=$ $\|\psi \phi\|_{m}$ for all $\phi \in M\left(\mathscr{E} ; \mathbf{R}^{n}\right)$ and all $\psi \in M(\mathscr{E} ; \mathbf{R})$. Let $\overline{\mathscr{S}}$ be the set of simple functions relative to the sequence $\left(\bar{O}_{k}\right)_{k \geqslant 1}$ introduced above. We have

$$
\begin{aligned}
\|\psi\|_{\phi \bullet m} & =\sup _{|\xi| \leqslant|\psi|, \xi \in \overline{\mathscr{S}}}\|(\phi \bullet m)(\xi)\|=\sup _{|\xi| \leqslant|\psi|, \xi \in \overline{\mathscr{S}}}\left\|\int(\phi \xi) \cdot \mathrm{d} m\right\| \\
& =\sup _{|\xi| \leqslant|\psi|, \phi \xi \in L^{1}(m)}\left\|\int(\phi \xi) \cdot \mathrm{d} m\right\|=\sup _{|\xi| \leqslant 1,(\xi \psi \phi) \in L^{1}(m)}\left\|\int(\xi \psi \phi) \cdot \mathrm{d} m\right\| \\
& =\|\psi \phi\|_{m},
\end{aligned}
$$

where the third equality follows using the DCT of the $m^{i}$ 's.

Since $1 \in L^{1}(\phi \bullet m)$ if and only if $\phi \bullet m$ extends to a finite $F$-valued measure on $(E, \mathscr{E})$, the vector space property of $L(m)$ together with the linearity of the integral is clear. The proof can now be completed by applying the DCT of the one-dimensional $\sigma$-finite measure $\phi \bullet m$.

\section{REFERENCES}

1. Barndorff-Nielsen O.E., Schmiegel J. Ambit processes: with applications to turbulence and tumour growth. - Stochastic Analysis and Applications. Berlin: Springer, 2007, p. 93-124. (Abel Symp., v. 2.)

2. Barndorff-Nielsen O.E., Schmiegel J. A stochastic differential equation framework for the timewise dynamics of turbulent velocities. - Теория вероятн. и ее примен., 2007, т. 52 , в. 3 , с. $541-561$.

3. Barndorff-Nielsen O.E., Schmiegel J. Brownian semistationary processes and volatility/intermittency. - Advanced Financial Modelling. Berlin: de Gruyter, 2009, p. 125. (Radon Ser. Comput. Appl. Math., v. 8.)

4. Basse-O'Connor A., Graversen S.-E., Pedersen J. Martingale-type processes indexed by the real line. - ALEA Lat. Am. J. Probab. Math. Statist., 2010, v. 7, p. 117-137.

5. Basse-O'Connor A., Graversen S.-E., Pedersen J. Some classes of proper integrals and generalized Ornstein-Uhlenbeck processes. - Lecture Notes in Math., 2012, v. 2046, p. 61-74.

6. Basse-O'Connor A., Graversen S.-E., Pedersen J. A unified approach to stochastic integration on the real line. - Thiele Research Report № 2010-08; http://www.imf.au.dk/publication/publid/880.

7. Behme A., Lindner A., Maller R. A. Stationary solutions of the stochastic differential equation $d V_{t}=V_{t-} d U_{t}+d L_{t}$ with Lévy noise. - Stochastic Process. Appl., 2011, v. 121, № 1, p. 91-108.

8. Bichteler $K$. Measures with values in non-locally convex spaces. - Lecture Notes in Math., 1976, v. 541, p. 277-285.

9. Bichteler K. Stochastic integration and $L^{p}$-theory of semimartingales. - Ann. Probab., 1981, v. 9, № 1, p. 49-89.

10. Bichteler K., Jacod J. Random measures and stochastic integration. - Lecture Notes in Control and Inform. Sci., 1983, v. 49, p. 1-18.

11. Cherny A., Shiryaev A. On stochastic integrals up to infinity and predictable criteria for integrability. - Lecture Notes in Math., 2005, v. 1857, p. 165-185. 
12. Dellacherie C., Meyer P.-A. Probabilities and Potential. B: Theory of Martingales. Amsterdam: North-Holland, 1982, 463 p. (North-Holland Math. Stud., v. 72.)

13. Doob J. L. Stochastic Processes. New York: Wiley, 1990, 654 p.

14. Emery M. Une topologie sur l'espace des semimartingales. - Lecture Notes in Math., 1979 , v. 721 , p. $260-280$.

15. Emery $M$. A generalization of stochastic integration with respect to semimartingales. - Ann. Probab., 1982, v. 10, № 3, p. 709-727.

16. Jacod J., Protter P. Time reversal on Lévy processes. - Ann. Probab., 1988, v. 16, № 2 , p. $620-641$.

17. Jacod J., Shiryaev A.N. Limit Theorems for Stochastic Processes. Berlin: SpringerVerlag, 2003, 661 p. (Grundlehren Math. Wiss., v. 288.)

18. Kwapień S., Woyczyński W. A. Semimartingale integrals via decoupling inequalities and tangent processes. - Probab. Math. Statist., 1991, v. 12, № 2, p. 165-200.

19. Kwapień S., Woyczyński W.A. Random Series and Stochastic Integrals: Single and Multiple. Boston: Birkhäuser, 1992, 360 p.

20. Lenglart E. Relation de domination entre deux processus. - Ann. Inst. H. Poincaré Sect. B (N.S.), 1977, v. 13, № 2, p. 171-179.

21. Mémin J. Espaces de semi martingales et changement de probabilité. - Z . Wahrscheinlichkeitstheor. verw. Geb., 1980, v. 52, № 1, p. 9-39.

22. Musielak J. Orlicz spaces and modular spaces. - Lecture Notes in Math., 1983, v. 1034, p. 1-222.

23. Rajput B.S., Rosiński J. Spectral representations of infinitely divisible processes. Probab. Theory Related Fields, 1989, v. 82, № 3, p. 451-487.

24. Rolewicz S. Metric Linear Spaces. Dordrecht: Reidel, 1985, 459 p. (Mathematics and its Applications (East European Ser.), v. 20.)

25. Rosiński J., Woyczyński W. A. On Itô stochastic integration with respect to $p$-stable motion: inner clock, integrability of sample paths, double and multiple integrals. Ann. Probab., 1986, v. 14, № 1, p. 271-286.

26. Ryll-Nardzewski C., Woyczyński W. A. Bounded multiplier convergence in measure of random vector series. - Proc. Amer. Math. Soc., 1975, v. 53, № 1, p. 96-98.

27. Sato K. Lévy Processes and Infinitely Divisible Distributions. Cambridge: Cambridge Univ. Press, 1999, 486 p. (Cambridge Stud. Adv. Math., v. 68.)

28. Schwartz L. Les semi-martingales formelles. - Lecture Notes in Math., 1981, v. 850, p. $413-489$.

29. Talagrand $M$. Les mesures vectorielles à valeurs dans $L^{0}$ sont bornées. - Ann. Sci. École Norm. Sup. (4), 1981, v. 14, № 4, p. 445-452. 